\title{
Rigorous Bounds on the Fast Dynamo Growth Rate Involving Topological Entropy
}

\author{
I. Klapper ${ }^{1, \star}$, L.S. Young ${ }^{2, \star \star}$ \\ 1 Program in Applied Mathematics, University of Arizona, Tucson, AZ 85721, USA \\ 2 Department of Mathematics, University of California, Los Angeles, CA 90024, USA
}

Received: 31 May 1994

\begin{abstract}
The fast dynamo growth rate for a $C^{k+1}$ map or flow is bounded above by topological entropy plus a $1 / k$ correction. The proof uses techniques of random maps combined with a result of Yomdin relating curve growth to topological entropy. This upper bound implies the following anti-dynamo theorem: in $C^{\infty}$ systems fast dynamo action is not possible without the presence of chaos. In addition topological entropy is used to construct a lower bound for the fast dynamo growth rate in the case $R_{m}=\infty$.
\end{abstract}

\section{The Kinematic Fast Dynamo Problem}

Magnetic dynamo theory involves the study of the generation of magnetic field in astrophysical objects such as planets and stars. One star of particular interest, of course, is the sun, which exhibits vigorous magnetic field activity on time scales much shorter than the magnetic diffusive time. Kinematic fast dynamo theory attempts to gain some understanding of the non-diffusive processes that might be involved by addressing the question of what sort of fluid motions can induce exponential growth of magnetic field at high magnetic Reynolds number. This is one of a large class of singular problems with important physical implications for which there is a need for a better understanding of the limiting behavior of complicated processes. Natural questions that arise are: what if any relation holds between the limiting and singular limit solutions, and what information about the limiting process can be gained from the singular limit problem? In this paper we consider a conjectured growth rate bound of Finn and Ott (1988) based on stretching properties of the fluid flow and prove a slightly generalized version of it.

The equations of dynamo theory are those of incompressible MHD (see Roberts (1967)). Denoting the magnetic field by $\mathbf{B}$ and the fluid velocity by $\mathbf{u}$ the magnetic

\footnotetext{
$\star$ This author is supported by an NSF postdoctoral fellowship

$\star \star$ This author is partially supported by an NSF grant
} 
induction equation

$$
\frac{\partial \mathbf{B}}{\partial t}+\mathbf{u} \cdot \nabla \mathbf{B}=\mathbf{B} \cdot \nabla \mathbf{u}+\frac{1}{R_{m}} \nabla^{2} \mathbf{B}, \quad \nabla \cdot \mathbf{B}=0
$$

can be derived from Ohm's law and the Maxwell equations (using the standard MHD approximation of neglecting the displacement current $\partial \mathbf{E} / \partial t) . R_{m}$, the magnetic Reynolds number, is a dimensionless parameter measuring the relative strength of advective to diffusive processes. Requiring $\nabla \cdot \mathbf{u}=0$ and setting the fluid density $\rho=1$ the fluid momentum equation is

$$
\frac{\partial \mathbf{u}}{\partial t}+\mathbf{u} \cdot \nabla \mathbf{u}=-\nabla p+\frac{1}{M_{A}}(\nabla \times \mathbf{B}) \times \mathbf{B}+\frac{1}{R} \nabla^{2} \mathbf{u},
$$

where $M_{A}$, the Alfven Mach number, is a dimensionless parameter measuring the typical balance between fluid and magnetic energy and $R$, the fluid Renolds number, is a dimensionless parameter measuring the typical balance between fluid advection and diffusion. Equations (1) and (2) are supplemented by the appropriate boundary and initial conditions.

We will make the kinematic dynamo approximation of setting $M_{A}=\infty$ (i.e., the magnetic energy is assumed small). As the Lorentz force $(\nabla \times \mathbf{B}) \times \mathbf{B}$ is quadratic in $\mathbf{B}$ this may perhaps be justified as a linearization of (2) around $\mathbf{B}=0$. Then the fluid momentum equation (2) is decoupled from the magnetic field and so the fluid velocity may be considered to be prescribed independently of $\mathbf{B}$ (thus the term kinematic). Equation (1) is now a linear equation for $\mathbf{B}$. The object of kinematic dynamo theory is to find linear dynamo instabilities, that is, to determine for a given $R_{m}$ which if any choices of $\mathbf{u}$ result in exponential growth of $\mathbf{B}$ for some initial conditions $\mathbf{B}_{0}$. For our purposes, given $\mathbf{u}(\mathbf{x}, t)$ we define kinematic dynamo action to occur when

$$
p\left(R_{m}\right) \equiv \lim _{T \rightarrow \infty} \frac{1}{T} \ln \int_{V}\left|\mathbf{B}\left(\mathbf{x}, T ; R_{m}\right)\right| d \mathbf{x}>0,
$$

where $V$ is the region containing magnetic field.

This paper addresses the kinematic fast dynamo problem (Vainshtein and Zel'dovich (1972)). A velocity field is defined to be a kinematic fast dynamo if

$$
\lim _{R_{m} \rightarrow \infty} p\left(R_{m}\right)>0 \text {. }
$$

(We assume that this limit exists; in principle one might instead use lim inf or lim sup.) Motivation for this definition comes from the solar magnetic field which shows activity (e.g. the 22 year solar cycle) on time scales much shorter than the diffusive time scale $\left(\sim 10^{10}\right.$ years $)$. Hence the solar dynamo apparently functions in some sense independently of diffusion. The object of kinematic fast dynamo theory is to characterize velocity fields that support dynamo action without direct use of diffusion. Definition (4) states roughly that dynamo activity should not cease in the limit of the diffusive term becoming small compared to the advective one.

\section{Statement of Result and Discussion}

Finn and Ott (1988) proposed that the kinematic fast dynamo growth rate of a steady or periodic flow is bounded above by the topological entropy of the flow. A proof in the $C^{\infty}$ case was announced by Vishik (1992). In this paper we prove 
a more general result using in a simple way standard techniques from dynamical systems.

Theorem 1. Let $\mathbf{u}$ and $\mathbf{B}_{0}$ be divergence free vector fields supported on a compact domain $\mathscr{D} \subset R^{d}$. We assume that $\mathbf{u}$ is of class $C^{k+1}$ and $\mathbf{B}_{0}$ is $C^{k}$ for some $k \geqq 2$. Let $\mathbf{f}$ be the time 1 map of the flow generated by $\mathbf{u}$, and let $\mathbf{B}\left(\mathbf{x}, t ; R_{m}\right)$ be the solution of Eq. (1) with initial conditions $\mathbf{B}_{0}(\mathbf{x})$. Then

$$
\limsup _{R_{m} \rightarrow \infty} \limsup _{n \rightarrow \infty} \frac{1}{n} \ln \int_{V}\left|\mathbf{B}\left(\mathbf{x}, n ; R_{m}\right)\right| d \mathbf{x} \leqq h(\mathbf{f})+\frac{r(\mathbf{f})}{k}
$$

where $h(\mathbf{f})$ is the topological entropy of $\mathbf{f}$ (restricted to $\mathscr{D})$ and

$$
r(\mathbf{f}) \equiv \lim _{n \rightarrow \infty} \frac{1}{n} \ln \max _{\mathbf{x} \in D}\left\|D \mathbf{f}^{n}(\mathbf{x})\right\|
$$

This upper bound is also valid for $R_{m}=\infty$.

None of the arguments to be presented depend on dimension (of course the higher dimension are not physically relevant). We also note that a number of conditions in Theorem 1 are easily relaxed or modified. For example, $\mathbf{u}$ may be time periodic, and $R^{d}$ can be replaced by $d$-dimensional torus (in which case, additionally, $\mathbf{B}_{0}$ need only be continuous), etc. See Sect. 7.

We state separately a version of Theorem 1 for maps, since maps have become popular models for fast dynamos (e.g. Bayly and Childress (1988), Finn and Ott (1988), Gilbert (1993), Soward (1994)). Let $\mathbf{f}$ be a diffeomorphism of $R^{d}$ and define a velocity field $\mathbf{u}_{\mathrm{f}}=\sum_{n=-\infty}^{\infty}(\delta(t-n) \mathbf{f})$. Then at $R_{m}=\infty$, Eq. (1) has the formal solution

$$
\mathbf{B}(\mathbf{x}, n+1)=\left(\mathbf{f}_{*} \mathbf{B}\right)(\mathbf{x}, n)=\nabla \mathbf{f}\left(\mathbf{f}^{-1}(\mathbf{x})\right) \cdot \mathbf{B}\left(\mathbf{f}^{-1}(\mathbf{x}), n\right) .
$$

This is the Cauchy solution, or in the language of dynamical systems, the pushforward of $\mathbf{B}$. Now let $\left\{\rho_{\varepsilon}, \varepsilon \geqq 0\right\}$ be a family of probability densities on $R^{d}$ with $\rho_{\varepsilon}$ tending weakly to $\rho_{0}=\delta(0)$, the delta function at 0 , as $\varepsilon \rightarrow 0$.

Theorem 1'. Let $\mathbf{f}$ be a $C^{k}$ volume preserving diffeomorphism of $R^{d}$ with $\mathbf{f}=\mathbf{I d}$ outside a compact domain $\mathscr{D}$, and let $\mathbf{B}_{0}$ be as in Theorem 1 . For $n=1,2, \ldots$, we define $\mathbf{B}_{n+1} \equiv \mathbf{B}\left(\mathbf{x}, n+1 ; R_{m}\right)$ to be the convolution of $\mathbf{f}_{*} \mathbf{B}_{n}$ with $\rho_{R_{m}^{-1}}$. Then the conclusions of Theorem 1 hold.

The case of particular interest here is when $\rho_{\varepsilon}$ is a $d$-dimensional Gaussian density with isotropic variance $\varepsilon$. Then $\mathbf{B}_{n}$ is the formal solution to (1) with $\mathbf{u} \equiv \mathbf{u}_{\mathbf{f}}$ and $R_{m}=\varepsilon^{-1}$ as above.

Certain remarks are in order here. First, positive topological entropy implies chaos in a topological sense - this will be further discussed in the next section. Thus Theorems 1 and $1^{\prime}$ are essentially anti-dynamo theorems; if $\mathbf{u}(\mathbf{x})($ or $\mathbf{f}(\mathbf{x}))$ is $C^{\infty}$ and not chaotic then $\mathbf{u}(\mathbf{x})$ ( or $\mathbf{f}(\mathbf{x})$ ) is not a fast dynamo. Second, in the case $k=\infty$ the upper bound can be achieved by Anosov systems (Arnol'd et al. (1981), Bayly (1986), Vishik (1989), Collet (1992), Gilbert (1993), Oseledets (1993)). Next, the two well known fast dynamo flows of Soward (1987) and Gilbert (1988) are not $C^{k}, k>2$ and are not covered by Theorem 1; singularities in the field of Jacobian 
matrices are needed in an essential way. (In such cases the methods of this paper cannot be applied.) Lastly, in addition to the result of Vishik mentioned at the beginning of the section, we mention that Oseledets (1993) has derived an exact growth rate for $C^{\infty}$ maps on the 2 torus. His methods rely on special properties of 2-dimensional magnetic fields (Zel'dovich (1957)).

The proofs of Theorems 1 and $1^{\prime}$ are based on three basic ideas. First, magnetic field lines at $R_{m}=\infty$ are material curves, and the magnetic field grows because of stretching of these material curves. Thus the field growth rate is bounded above by the material curve growth rate, at least for $R_{m}=\infty$. Here we emphasize that we refer to the growth rate of curves of finite extent. As noted by Finn and Ott (1988) infinitesimal line elements, i.e., tangent vectors, grow more slowly in general, and their growth rate does not necessarily bound the magnetic field growth rate. The second idea, due to Yomdin (1987), is that when a map is iterated the growth rate of a material curve is bounded above by topological entropy plus a possible correction due to a lack of smoothness. This bound can be understood heuristically by discretizing a section of curve with a large number of points. The growth rate of the curve is approximated by the growth rate of the number of points needed to resolve the curve to a given tolerance, which in turn is, roughly speaking, bounded by the topological entropy (see Sect. 3). But also, between the fine discretization, lack of smoothness in the map or flow can cause the curve to locally "crinkle" in such a way as to increase the growth rate by as much as $r / k$. The third basic idea is to exploit the fact that diffusion of the magnetic field can be accomplished by adding noise to the underlying dynamical system. This allows us to consider random maps and to use a randomized version of Yomdin's arguments. We show that a small amount of noise does not increase the topological entropy. Thus, in the limit, the same upper bound on magnetic field growth holds. (The introduction of noise as an analytical tool for studying fast dynamos can be found in Molchanov et al. (1985) and Klapper (1993); it has also found use as a numerical method (Klapper (1992)).)

To further highlight the relationship between topological entropy, the growth rate of material curves, and the fast dynamo problem, we prove the following lower bound for magnetic field growth at $R_{m}=\infty$ :

Theorem 2. Let $\mathscr{D}$ be a compact domain in $R^{d}$ and let $\mathbf{f}: \mathscr{D} \rightarrow \mathscr{D}$ be a $C^{1, \alpha}(\alpha>0)$ volume preserving diffeomorphism of $\mathscr{D}$. Then $\forall q \geqq d-1(\forall q \geqq d-2$ if $\mathbf{f}$ is the time 1 map of a flow) we have

$$
\sup _{\mathbf{B}_{0}} \liminf _{n \rightarrow \infty} \frac{1}{n} \ln \int_{\mathscr{D}}|\mathbf{B}(\mathbf{x}, n ; \infty)|^{q} d \mathbf{x} \geqq h(\mathbf{f}),
$$

where $h(\mathbf{f})$ is the topological entropy of $\mathbf{f}$ and the supremum is taken over all $C^{\infty}$ divergence free vector fields $\mathbf{B}_{0}$ with compact support.

We remark again that flows are considered steady; a periodic flow in dimension $d$ can be regarded as a steady flow in dimension $d+1$.

The organization of the paper is as follows. Section 3 provides a brief introduction to the relevant facts of topological entropy. In Sect. 4 we prove Theorems 1 and $1^{\prime}$ in the special case that $R_{m}=\infty$. While not of direct physical significance this case provides an opportunity to emphasize the first two basic ideas of the proof. Section 5 extends the results of Sect. 4 for maps from $R_{m}=\infty$ to $R_{m} \rightarrow \infty$ completing the proof of Theorem $1^{\prime}$. Section 6 is concerned with those technical details necessary to extend our methods from maps to flows and thus finish the proof of 
Theorem 1. Section 7 contains remarks concerning the range of applications of the presented results to fast dynamos and related problems, and Sect. 8, which is independent of the rest of the paper, contains a proof of Theorem 2 , the $R_{m}=\infty$ lower bound.

The authors thank J. Watkins for conversations related to Sect. 6 and A. Gilbert for helpful comments.

\section{Topological Entropy}

For the convenience of readers not familiar with this subject we present here two equivalent definitions of topological entropy. For further details and background on this section see Walters (1982). The first definition, due to Adler et al. (1965), is based on open covers (hence the adjective topological). Let $\mathscr{D}$ be a compact topological space, and let $\alpha$ be an open cover of $\mathscr{D}$. Define $H(\alpha)$, the topological entropy of $\alpha$, by $H(\alpha) \equiv \ln N(\alpha)$, where $N(\alpha)$ is the smallest cardinality of any finite subcover of $\alpha$. Given $\mathbf{f}: \mathscr{D} \rightarrow \mathscr{D}$ a continuous map, $h(\mathbf{f}, \alpha)$, the entropy of $\mathbf{f}$ relative to $\alpha$, is defined to be

$$
h(\mathbf{f}, \alpha)=\lim _{n \rightarrow \infty} \frac{1}{n} H\left(\bigvee_{l=0}^{n-1} \mathbf{f}^{-i} \alpha\right) .
$$

Here $\mathbf{f}^{-1} \alpha$ is the open cover consisting of those sets $\mathbf{f}^{-1} A$ such that $A$ is an element of $\alpha$, and the join $\alpha \vee \beta$ of two open covers $\alpha$ and $\beta$ is the open cover consisting of all sets of the form $A \cap B$ with $A \in \alpha, B \in \beta$. Finally, the topological entropy of the map $\mathbf{f}$ is defined by

$$
h(\mathbf{f})=\sup _{\alpha} h(\mathbf{f}, \alpha) .
$$

The significance of positive topological entropy can be intuitively understood in the following manner. Consider a cover $\alpha$ with a finite number of elements and let $\mathcal{O}$ be an element of $\bigvee_{i=0}^{n-1} \mathbf{f}^{-l} \alpha$. Then $\mathcal{O}$ is of the form $A_{i_{1}} \cap \mathbf{f}^{-1} A_{l_{2}} \cap \cdots \cap \mathbf{f}^{-(n-1)} A_{l_{n-1}}$ for some $A_{i_{1}} \in \alpha$, i.e., $\mathbf{x} \in \mathcal{O}$ iff $\mathbf{x} \in A_{l_{1}}, \mathbf{f}(\mathbf{x}) \in A_{i_{2}}, \ldots, \mathbf{f}^{n-1}(\mathbf{x}) \in A_{l_{n-1}}$. We think of the trajectories of $\mathbf{x}$ and $\mathbf{y}$ as indistinguishable with respect to $\alpha$ if $\mathbf{f}^{l}(\mathbf{x})$ and $\mathbf{f}^{i}(\mathbf{y})$ fall in the same element of $\alpha \forall i$. The number $N\left(\bigvee_{0}^{n-1} \mathbf{f}^{-i} \alpha\right)$ then measures the number of trajectories of length $n$ that are pairwise distinguishable with respect to the labeling system given by $\alpha$, and positive entropy tells us that this number grows exponentially with $n$. We take this to be an indicator of chaos. Positive topological entropy in differentiable dynamical systems is often detected through the presence of horseshoes (e.g. using Melnikov methods). In dimension 2, it has in fact been shown that essentially all of the entropy of a system is carried by horseshoes (Katok (1980)).

Next we give a second definition of topological entropy due to Bowen (1971) for a continuous map $\mathbf{f}: \mathscr{D} \rightarrow \mathscr{D}$, where $(\mathscr{D}, d)$ is a metric space. We again assume that $\mathscr{D}$ is compact (although this definition can be adapted to non-compact situations). First we define a new metric $d_{n}$, dependent on $\mathbf{f}$, by

$$
d_{n}(\mathbf{x}, \mathbf{y})=\max _{0 \leqq i \leqq n} d\left(\mathbf{f}^{i}(\mathbf{x}), \mathbf{f}^{l}(\mathbf{y})\right) .
$$

Now, given $\varepsilon>0$ and an integer $n \geqq 0$ we say that a set $S \subseteq \mathscr{D}(n, \varepsilon)$-spans $\mathscr{D}$ if $\forall \mathbf{y} \in \mathscr{D} \exists \mathbf{x} \in S$ such that $d_{n}(\mathbf{x}, \mathbf{y}) \leqq \varepsilon$. In other words for any $\mathbf{y} \in \mathscr{D}$ there is some 
$\mathbf{x} \in S$ such that the trajectories of $\mathbf{x}$ and $\mathbf{y}$ over $n$ iterations of $\mathbf{f}$ are indistinguishable to resolution $\varepsilon$. Let $\tau_{n}(\varepsilon)$ be the smallest possible cardinality for any $(n, \varepsilon)$ spanning set. Then $\tau_{n}(\varepsilon)$ measures the number of different trajectories of length $n$ to resolution $\varepsilon$. In terms of the metric $d_{n}, \tau_{n}(\varepsilon)$ is the minimum number of balls $b(\mathbf{x}, n ; \varepsilon)$ of radius $\varepsilon$ defined by

$$
b(\mathbf{x}, n ; \varepsilon)=\left\{\mathbf{y}: d_{n}(\mathbf{x}, \mathbf{y})<\varepsilon\right\}
$$

needed to cover $\mathscr{D}$. We now define the topological entropy to be

$$
h(\mathbf{f})=\lim _{\varepsilon \rightarrow 0} \limsup _{n \rightarrow \infty} \frac{1}{n} \ln \tau_{n}(\varepsilon) \text {. }
$$

In the previous language, this is the growth rate in $n$ of the number of distinct trajectories measured at finer and finer resolution $\varepsilon$. For compact spaces it is easily shown that for any metric $d$ that induces a given topology definition (8) is independent of that metric and equivalent to the open cover definition.

We now state without proof (see Walters (1982)) some properties of topological entropy that will be of use later:

Property 1. $h\left(\mathbf{f}^{m}\right)=m h(\mathbf{f})$.

The quantity $\operatorname{diam}(\alpha)$, the diameter of $\alpha$, is defined to be the supremum of the diameters of the elements of $\alpha$.

Property 2. If $\alpha_{n}$ is a sequence of open covers of $\mathscr{D}$ with $\operatorname{diam}\left(\alpha_{n}\right) \rightarrow 0$ as $n \rightarrow \infty$, then

$$
h(\mathbf{f})=\lim _{n \rightarrow \infty} h\left(\mathbf{f}, \alpha_{n}\right)
$$

\section{Property 3.}

$$
\tau_{n}(\varepsilon) \leqq \operatorname{card}\left(\bigvee_{i=0}^{n} \mathbf{f}^{-i} \alpha\right)
$$

where $\operatorname{diam}(\alpha)<\varepsilon$.

The notion of entropy generalizes to random maps. Let $C(\mathscr{D}, \mathscr{D})$ be the set of continuous maps from $\mathscr{D}$ to $\mathscr{D}$ and let $v$ be a probability measure on $C(\mathscr{D}, \mathscr{D})$. Define $\mathbf{g}^{(n)}=\mathbf{g}_{n} \circ \cdots \circ \mathbf{g}_{1} \circ \mathbf{g}_{0}$, where $\mathbf{g}_{0}=I d$ and $\mathbf{g}_{i} \in C(\mathscr{D}, \mathscr{D})$ (and define $\mathbf{g}^{-(n)}=$ $\left.\mathbf{g}_{1}^{-1} \circ \mathbf{g}_{2}^{-1} \circ \cdots \circ \mathbf{g}_{n}^{-1}\right)$. We note that the definitions (6) and (8) generalize to sequences $\omega=\left\{\mathbf{g}_{0}, \mathbf{g}_{1}, \mathbf{g}_{2}, \ldots\right\}$ chosen iid with distribution $v$, where $\mathbf{f}^{i}$ is replaced by $\mathbf{g}^{(i)}$ and $\tau_{n}$ is calculated using balls $b(\mathbf{x}, n ; \varepsilon)$ defined by

$$
b(\mathbf{x}, n ; \varepsilon)=\left\{\mathbf{y}: d\left(\mathbf{g}^{(l)}(\mathbf{x}), \mathbf{g}^{(l)}(\mathbf{y})\right)<\varepsilon, 0 \leqq i \leqq n\right\} .
$$

In particular the limits exist and are nonrandom (i.e., independent of $\omega$ ) (Kifer (1986)).

When working with random maps of $R^{d}$, problems could arise with the use of topological entropy since $R^{d}$ is not compact. Note however that Bowen's definition continues to make sense if we limit our attention to orbits that originate from a compact set $\mathscr{D}$. When considering (non-random) maps $\mathbf{f}$ defined on all of $R^{d}$ but with $\mathbf{f}(\mathbf{x})=\mathbf{x} \forall \mathbf{x} \notin \mathscr{D}$, we denote the topological entropy of $\mathbf{f}$ restricted to $\mathscr{D}$ by $h(\mathbf{f} \mid \mathscr{D})$. 


\section{Growth of Magnetic Field at $\boldsymbol{R}_{m}=\infty$}

We begin with the $R_{m}=\infty$ case of Theorem 1 (which, as will become evident, is identical to the $R_{m}=\infty$ case of Theorem $1^{\prime}$ ). Let $\mathbf{f}$ be the time 1 map of the flow $\mathbf{u}$, and let the initial conditions $\mathbf{B}_{0}$ be a $C^{k}$ vector field supported on $\mathscr{D}$. When $R_{m}=\infty$, the solution to Eq. (1) is given by

$$
\mathbf{B}(\mathbf{x}, n)=\mathbf{J}\left(\mathbf{f}^{-n}(\mathbf{x}), n\right) \mathbf{B}_{0}\left(\mathbf{f}^{-n}(\mathbf{x})\right),
$$

the Cauchy solution, where $\mathbf{J}\left(\mathbf{f}^{-n}(\mathbf{x}), n\right)$ is the Jacobian matrix of the time $n$ map of the flow evaluated along the trajectory of the point $\mathbf{f}^{-n}(\mathbf{x})$. We will abbreviate $\mathbf{J}(\cdot, n)$ by $D \mathbf{f}^{n}(\cdot)$ (and in general $D^{l}$ denotes the tensor of $\ell^{\text {th }}$ order derivatives). Since for $R_{m}=\infty$ the magnetic field is a material vector field, it is reasonable to suggest that the field growth rate $p\left(R_{m}=\infty\right)$ is related to the growth rate of material curves. This is the subject of Claim 1.

Define a $C^{k}$ curve $\boldsymbol{\sigma}$ in $\mathscr{D}$ to be a $C^{k}$ map $\boldsymbol{\sigma}:[0,1] \rightarrow \mathscr{D}$ and let $\|\boldsymbol{\sigma}\|_{k} \equiv$ $\max _{1 \leqq l \leqq k}\left\|D^{l} \boldsymbol{\sigma}\right\|$. We will often confuse $\boldsymbol{\sigma}$ with its image and use $\ell(\boldsymbol{\sigma})$ to denote the length of the image.

Claim 1. If $R_{m}=\infty$, then for every $C^{k}$ initial condition $\mathbf{B}_{0}$,

$$
\limsup _{n \rightarrow \infty} \frac{1}{n} \ln \int_{\mathscr{C}}|\mathbf{B}(\mathbf{x}, n)| d \mathbf{x} \leqq \limsup _{n \rightarrow \infty} \frac{1}{n} \ln \left[\sup _{\sigma} \ell\left(\mathbf{f}^{n} \sigma\right)\right],
$$

where the supremum is taken over $C^{k}$ curves $\boldsymbol{\sigma}:[0,1] \rightarrow \mathscr{D}$ with $\|\boldsymbol{\sigma}\|_{k} \leqq 1$.

Proof. Using the Cauchy solution this is equivalent to showing that

$$
\limsup _{n \rightarrow \infty} \frac{1}{n} \ln \int_{\mathscr{L}}\left|D \mathbf{f}^{n} \mathbf{B}_{0}(\mathbf{x})\right| d \mathbf{x} \leqq \limsup _{n \rightarrow \infty} \frac{1}{n} \ln \left[\sup _{\sigma} \ell\left(\mathbf{f}^{n} \sigma\right)\right] \text {. }
$$

Choose a $C^{k}$ vector field $\mathbf{B}_{0}$ with support contained in $\mathscr{D}$. The natural way to demonstrate this inequality would be as follows: divide $\mathscr{D}$ into a number of flux tubes (or flow boxes) of $\mathbf{B}_{0}$ and track the stretching rate of these tubes. Having done this we can bound the growth rate of the magnetic field by the maximum stretching rate of a flux tube, which is in turn bounded by the maximum growth rate of a material curve. Unfortunately, this argument is problematic because dividing $\mathbf{B}_{0}$ into flux tubes becomes difficult near null points of $\mathbf{B}_{0}$. Instead we will use the following trick to accomplish much the same plan: Let $\mathbf{X}$ be an arbitrary constant vector field on $R^{d}$ with $|\mathbf{X}| \geqq 100 \max \left|\mathbf{B}_{0}(\mathbf{x})\right|$. Then neither $\mathbf{X}$ nor $\mathbf{B}_{0}-\mathbf{X}$ has null points and

$$
\begin{aligned}
& \limsup _{n \rightarrow \infty} \frac{1}{n} \ln \int_{\mathscr{Q}}\left|D \mathbf{f}^{n} \mathbf{B}_{0}(\mathbf{x})\right| d \mathbf{x} \\
& \quad \leqq \limsup _{n \rightarrow \infty} \frac{1}{n} \ln \left(\int_{\mathscr{D}}\left|D \mathbf{f}^{n} \mathbf{X}(\mathbf{x})\right| d \mathbf{x}+\int_{\mathscr{Z}}\left|D \mathbf{f}^{n}\left(\mathbf{B}_{0}-\mathbf{X}\right)(\mathbf{x})\right| d \mathbf{x}\right) \\
& \quad=\max \left\{\limsup _{n \rightarrow \infty} \frac{1}{n} \ln \int_{\mathscr{D}}\left|D \mathbf{f}^{n} \mathbf{X}(\mathbf{x})\right| d \mathbf{x}, \limsup _{n \rightarrow \infty} \frac{1}{n} \ln \int_{\mathscr{D}}\left|D \mathbf{f}^{n}\left(\mathbf{B}_{0}-\mathbf{X}\right)(\mathbf{x})\right| d \mathbf{x}\right\} .
\end{aligned}
$$

In fact, both $\mathbf{X}$ and $\mathbf{B}_{0}-\mathbf{X}$ point so uniformly in one direction that we may think of the active region $\mathscr{D}$ as being contained in a single flux tube. More precisely, let $\tilde{\mathbf{B}}_{0}=\mathbf{X}$ or $\mathbf{B}_{0}-\mathbf{X}$, and let $P$ be any hyperplane roughly perpendicular to $\mathbf{X}$. For 
$\mathbf{x} \in P$, let $\boldsymbol{\sigma}_{\mathbf{x}}$ be the integral curve of $\tilde{\mathbf{B}}_{0}$ with $\boldsymbol{\sigma}_{\mathbf{x}}(0)=\mathbf{x}$. Choose $r_{0}$ large enough so that $\mathscr{D}$ is contained in $\Gamma \equiv\left\{\mathbf{y}=\boldsymbol{\sigma}_{\mathbf{x}}(s)\right.$ for some $\mathbf{x} \in P$ and $\left.|s| \leqq r_{0}\right\}$. Then

$$
\begin{aligned}
& \underset{n \rightarrow \infty}{\limsup } \frac{1}{n} \ln \int_{\mathscr{D}}\left|D \mathbf{f}^{n} \tilde{\mathbf{B}}_{0}(\mathbf{x})\right| d \mathbf{x} \leqq \limsup _{n \rightarrow \infty} \frac{1}{n} \ln \int_{\Gamma}\left|D \mathbf{f}^{n} \tilde{\mathbf{B}}_{0}(\mathbf{x})\right| d \mathbf{x} \\
& \quad=\limsup _{n \rightarrow \infty} \frac{1}{n} \ln \int_{\Delta=\Gamma \cap P} \ell\left(\mathbf{f}^{n} \boldsymbol{\sigma}_{\mathbf{x}}\right) d F(\mathbf{x}) \leqq \limsup _{n \rightarrow \infty} \frac{1}{n} \ln \left(\sup _{\mathbf{x} \in \Delta} \ell\left(\mathbf{f}^{n} \sigma_{\mathbf{x}}\right)\right),
\end{aligned}
$$

where $d F$ is the flux (or transverse measure) of $\tilde{\mathbf{B}}_{0}$ across $\Delta$ and $\boldsymbol{\sigma}_{\mathbf{x}}$ is understood to be defined on $\left[-r_{0}, r_{0}\right]$. We here have used the fact that $\tilde{\mathbf{B}}_{0}$ is divergence free and thus the total flux is independent of the choice of cross-section.

To finish it remains to observe that the right side of inequality (10) remains unchanged if the supremum is taken over curves $\sigma$ defined on intervals of length $\leqq C$ and with $\|\boldsymbol{\sigma}\|_{k} \leqq C$ for a fixed $C$. This is true because by subdividing and reparametrizing, each $\boldsymbol{\sigma}$ can be decomposed into $\boldsymbol{\sigma}=\boldsymbol{\sigma}_{1} \cup \cdots \cup \boldsymbol{\sigma}_{p}$, where each $\boldsymbol{\sigma}_{i}$ is normalized according to the requirements of claim 1 .

Next we relate the growth rate of material curves to topological entropy following Yomdin.

Claim 2.

$$
\limsup _{n \rightarrow \infty} \frac{1}{n} \ln \left(\sup _{\boldsymbol{\sigma}} \ell\left(\mathbf{f}^{n} \boldsymbol{\sigma}\right)\right) \leqq h(\mathbf{f})+\frac{r(\mathbf{f})}{k},
$$

where $\sigma$ is as in Claim 1 and $r$ is defined as in the statement of Theorem 1.

Proof. This is a slightly stronger statement than that made by Yomdin (1987) but it follows immediately from Yomdin's proof. The idea is as follows: let

$$
b(\mathbf{x}, n ; \varepsilon)=\left\{\mathbf{y}: d\left(\mathbf{f}^{l}(\mathbf{x}), \mathbf{f}^{l}(\mathbf{y})\right)<\varepsilon, 0 \leqq i \leqq n\right\},
$$

so that $\tau_{n}(\varepsilon)$ is the smallest number of sets of this form needed to cover $\mathscr{D}$. Given a curve $\sigma$, let

$$
V(\mathbf{f}, \boldsymbol{\sigma} ; \varepsilon)=\sup _{\mathbf{x} \in \mathscr{D}} \ell\left[\mathbf{f}^{n} \boldsymbol{\sigma} \cap b(\mathbf{x}, n ; \varepsilon)\right]
$$

Then

$$
\ell\left(\mathbf{f}^{n} \boldsymbol{\sigma}\right) \leqq \tau_{n}(\varepsilon) \cdot V(\mathbf{f}, \boldsymbol{\sigma} ; \varepsilon) .
$$

In other words we distinguish between 2 types of growth. The growth rate of $\tau_{n}(\varepsilon)$, i.e., topological entropy, bounds the growth rate of $\ell\left(\mathbf{f}^{n} \boldsymbol{\sigma}\right)$ as detected up to resolution $\varepsilon$ (uniformly in $\boldsymbol{\sigma}$ ), whereas local crinkling-crinkling that occurs in smaller scales but which can in principle pile up exponentially fast -is measured by the growth rate of $V(\mathbf{f}, \boldsymbol{\sigma} ; \varepsilon)$. Yomdin proved that

$$
\lim _{\varepsilon \rightarrow 0} \limsup _{n \rightarrow \infty} \frac{1}{n} \ln \left[\sup _{\boldsymbol{\sigma}} V(\mathbf{f}, \boldsymbol{\sigma} ; \varepsilon)\right] \leqq \frac{r(\mathbf{f})}{k}
$$

if $\mathbf{f}$ is $C^{k}$. Appendix A contains an outline of his argument.

Claims (1) and (2) together prove the $R_{m}=\infty$ cases of Theorems 1 and $1^{\prime}$.

We close this section with a discussion of growth rates of finite material curves versus growth rates of infinitesimal line elements. Let $\sigma:[0,1] \rightarrow \mathscr{D}$ be such a 
curve, and suppose for the sake of argument that for almost every $s \in[0,1]$,

$$
\lim _{n \rightarrow \infty} \frac{1}{n} \ln \left|D \mathbf{f}^{n} \boldsymbol{\sigma}^{\prime}(s)\right|=\lambda \text {. }
$$

Integrating, we obtain

$$
\begin{aligned}
\lambda & =\lim _{n \rightarrow \infty} \frac{1}{n} \int_{0}^{1} \ln \left|D \mathbf{f}^{n} \boldsymbol{\sigma}^{\prime}(s)\right| d s \leqq \liminf _{n \rightarrow \infty} \frac{1}{n} \ln \int_{0}^{1}\left|D \mathbf{f}^{n} \boldsymbol{\sigma}^{\prime}(s)\right| d s \\
& =\liminf _{n \rightarrow \infty} \frac{1}{n} \ln \ell\left(\mathbf{f}^{n} \boldsymbol{\sigma}\right) .
\end{aligned}
$$

If the vectors $D \mathbf{f}^{n} \boldsymbol{\sigma}^{\prime}(s)$ converge to their eventual growth rate nonuniformly for different $s$, then the above inequality could be strict. This is in fact generally the case: metric entropy, which for volume preserving diffeomorphisms is the sum of the positive Lyapunov exponents, is almost always strictly smaller than the topological entropy. This means that the growth rate of a curve is often dominated by stretching at exceptional points.

As far as the dynamo problem goes, it has been observed that Lyapunov exponents do not necessarily bound the growth rates of magnetic fields. The proof of Theorem 2 will shed further light on how field growth rates are tied to growth rates of curves, which in turn are tied to the growth rates of infinitesimal line elements at certain exceptional points.

\section{Upper Bound on the Growth Rate for Maps in the Limit $\boldsymbol{R}_{\boldsymbol{m}} \rightarrow \infty$}

In this section we complete the proof of Theorem $1^{\prime}$. The main point of consideration is the growth rate of curves under the action of random maps most of which are small perturbations of $\mathbf{f}$. We show that in the zero-noise limit this growth rate has the same upper bound as that for growth of curves under the action of $\mathbf{f}$.

Denote by $\Omega$ the set of $C^{k}$ volume preserving diffeomorphisms on $R^{d}$ and let $\mathbf{f} \in$ $\Omega$ be such that $\mathbf{f}$ is equal to the identity outside of the compact domain $\mathscr{D}$. We consider a one parameter family of probability measures $v_{\eta}$ on $\Omega$ with $\eta \geqq 0$ such that

1. $\left\|D^{l} \mathbf{g}\right\| \leqq C, 1 \leqq l \leqq k$, for $v_{\eta}$ a.s. $\mathbf{g}$, where $C$ is a constant independent of $\eta$;

2. $\forall \delta>0$ we have $v_{n}\left\{\mathbf{g}:\|\mathbf{g}-\mathbf{f}\|_{C^{k}}<\delta\right\} \rightarrow 1$ as $\eta \rightarrow 0$.

(The $C^{k}$ norm of $\mathbf{h},\|\mathbf{h}\|_{C^{k}}$, is defined to be $\max \left\|D^{s} \mathbf{h}\right\|, 0 \leqq s \leqq k$, and should not be confused with the norm $\|\mathbf{h}\|_{k} \equiv \max \left\|D^{s} \mathbf{h}\right\|, 1 \leqq s \leqq k$ ). For a given $\mathbf{B}_{0}$ define $\mathbf{B}_{n, \eta}$ by

$$
\mathbf{B}_{n, \eta}=\int_{\Omega}\left(D \mathbf{g} \mathbf{B}_{n-1, \eta}\right) v_{\eta}(d \mathbf{g})=\int_{\Omega^{n}}\left(D \mathbf{g}^{(n)} \mathbf{B}_{0}\right) v_{\eta}^{n}\left(d \mathbf{g}_{1}, \ldots, d \mathbf{g}_{n}\right),
$$

where $\mathbf{g}^{(n)}=\mathbf{g}_{n} \circ \cdots \circ \mathbf{g}_{1}, v_{\eta}^{n}=v_{\eta} \times \cdots \times v_{\eta}$. The aim of this section is to prove the following:

Proposition 1. Let $v_{\eta}$ and $\mathbf{B}_{n, \eta}$ be defined as above. Then

$$
\limsup _{\eta \rightarrow 0} \limsup _{n \rightarrow \infty} \frac{1}{n} \ln \int_{R^{d}}\left|\mathbf{B}_{n, \eta}\right| d \mathbf{x} \leqq h(\mathbf{f})+\frac{r(\mathbf{f})}{k},
$$

where $h(\mathbf{f})$ is the topological entropy of $\mathbf{f}$ restricted to $\mathscr{D}$. 
To prove Theorem $1^{\prime}$, let $v_{\eta}$ be the distribution of $\mathbf{f}+\omega$, where $\omega$ is a $d$-dimensional vector distributed according to $\rho_{\eta}$. Then

$$
\mathbf{B}_{n, \eta}(\mathbf{x})=\int\left(D \mathbf{f} \mathbf{B}_{n-1, \eta}\right)\left(\mathbf{f}^{-1}(\mathbf{y})\right) \rho_{\eta}(\mathbf{y}-\mathbf{x}) d \mathbf{y}
$$

If in addition $\rho_{\eta}$ gives a distribution consisting of $d$ dimensional vectors with independent gaussian entries of variance $2 \eta$, then $\mathbf{B}_{n, \eta}(\mathbf{x})$ formally solves the magnetic induction equation (1) with the $\delta$-function flow defined in Sect. 2.

We begin the proof of Proposition 1 with the following observation:

$$
\int_{R^{d}}\left|\mathbf{B}_{n, \eta}\right| d \mathbf{x}=\int_{\mathscr{Z}}\left|\int_{\Omega^{n}} D \mathbf{g}^{(n)} \mathbf{B}_{0} d v_{\eta}^{n}\right| d \mathbf{x} \leqq \int_{\Omega^{n}}\left(\int_{\mathscr{Z}}\left|D \mathbf{g}^{(n)} \mathbf{B}_{0}\right| d \mathbf{x}\right) d v_{\eta}^{n}
$$

and, by identical reasoning to that used in the previous section,

$$
\begin{aligned}
\limsup _{n \rightarrow \infty} \frac{1}{n} \ln \int_{\Omega^{n}} \int_{\mathscr{D}}\left|D \mathbf{g}^{(n)} \mathbf{B}_{0}\right| d \mathbf{x} d v_{\eta}^{n} & \leqq \limsup _{n \rightarrow \infty} \frac{1}{n} \ln \int_{\Omega^{n}} \int_{\Gamma}\left|D \mathbf{g}^{(n)} \tilde{\mathbf{B}}_{0}\right| d \mathbf{x} d v_{\eta}^{n} \\
& =\limsup _{n \rightarrow \infty} \frac{1}{n} \ln \int_{\Omega^{n}}\left(\int_{\Delta} \ell\left(\mathbf{g}^{n} \sigma_{\mathbf{x}}\right) d F(\mathbf{x})\right) d v_{\eta}^{n},
\end{aligned}
$$

where $\Gamma$ is a flux tube of some vector field $\tilde{\mathbf{B}}_{0}$ (that depends only on $\mathbf{B}_{0}$ ) and $\Delta$ is a transverse section of $\Gamma$. Moreover we can interchange limits again to obtain

$$
\begin{aligned}
\int_{\Omega^{n}}\left(\int_{\Delta} \ell\left(\mathbf{g}^{(n)} \boldsymbol{\sigma}_{\mathbf{x}}\right) d F(\mathbf{x})\right) d v_{\eta}^{n} & =\int_{\Delta}\left(\int_{\Omega^{n}} \ell\left(\mathbf{g}^{(n)} \boldsymbol{\sigma}_{\mathbf{x}}\right) d v_{\eta}^{n}\right) d F(\mathbf{x}) \\
& \leqq F(\Delta) \sup _{\mathbf{x} \in \Delta} \int_{\Omega^{n}} \ell\left(\mathbf{g}^{(n)} \boldsymbol{\sigma}_{\mathbf{x}}\right) d v_{\eta}^{n}
\end{aligned}
$$

where $F(\Delta)$ is the flux of $\tilde{\mathbf{B}}_{0}$ through $\Delta$ (a constant). For the rest of the proof let us assume that $\mathscr{D}$ is enlarged to contain $\Gamma$ and that $\mathbf{f}=\mathbf{I d}$ in a neighborhood of the boundary of $\mathscr{D}$. The proof is then reduced to the following claim:

Claim 3. Given $\varepsilon_{0}>0, \exists \eta_{0}>0$ such that $\forall \eta<\eta_{0} \exists N$ such that $\forall n \geqq N$ and $\forall \boldsymbol{\sigma}:[0,1] \rightarrow \mathscr{D}$ with $\|\sigma\|_{k} \leqq 1$,

$$
\int \ell\left(\mathbf{g}^{n} \boldsymbol{\sigma}\right) v_{\eta}^{n}\left(d \mathbf{g}^{n}\right) \leqq e^{n\left(h(\mathbf{f})+r(\mathbf{f}) / k+5 \varepsilon_{0}\right)} .
$$

Proof of Claim 3.

Step 1. Preliminary choices.

(a) For each $\varepsilon>0$, let $\alpha_{\varepsilon}$ be a covering of $R^{d}$ by (round) balls of diameter $\leqq \varepsilon$. We assume that $\alpha_{\varepsilon}$ is uniformly spaced so that

(i) no element of $\alpha_{\varepsilon}$ intersects more than $C_{d}$ other elements of $\alpha_{\varepsilon}$, and

(ii) $\exists \ell(\varepsilon)>0$ such that $\forall \mathbf{x} \in R^{d}$, the $\ell(\varepsilon)$-ball centered at $\mathbf{x}$ is contained in some element of $\alpha_{\varepsilon}$ (i.e., $\ell(\varepsilon)$ is a Lebesgue number for $\alpha_{\varepsilon}$ ).

The subcollection $\left\{A \in \alpha_{\varepsilon}: A \cap \mathscr{D} \neq \emptyset\right\}$ will be denoted by $\alpha_{\varepsilon} \mid \mathscr{D}$. 
(b) We choose $\varepsilon>0$ such that

(i) $h\left(\mathbf{f} \mid \mathscr{D}, \alpha_{\varepsilon}\right)<h(\mathbf{f} \mid \mathscr{D})+\varepsilon_{0}$, and

(ii) if $\mathbf{g}_{\varepsilon}(\mathbf{x}) \equiv(1 / \varepsilon) \mathbf{g}(\mathbf{x} / \varepsilon)$, then for $v_{\eta}$-a.e. $\mathbf{g},\left\|D^{l} \mathbf{g}_{\varepsilon}\right\| \leqq\left\|D \mathbf{g}_{\varepsilon}\right\|$ for $l=1, \ldots, k$ ((i) is possible by Property 2 in Sect. 3, and (ii) is possible because of our first requirement on $v_{\eta}$ (see also Appendix A)).

(c) For a sequence $\left(\mathbf{g}_{0}, \ldots, \mathbf{g}_{n}\right)$ with $\mathbf{g}_{0}=\mathbf{I d}$, we will estimate $\ell\left(\mathbf{g}^{(n)} \boldsymbol{\sigma}\right)$ by

$$
\ell\left(\mathbf{g}^{(n)} \boldsymbol{\sigma}\right) \leqq \tau_{n}\left(\mathbf{g}^{(n)}, \varepsilon\right) \cdot V\left(\mathbf{g}^{(n)}, \boldsymbol{\sigma} ; \varepsilon\right)
$$

where $\tau_{n}\left(\mathbf{g}^{(n)}, \varepsilon\right)$ is the minimum number of sets of the form

$$
b\left(\mathbf{x}, \mathbf{g}^{(n)} ; \varepsilon\right)=\left\{\mathbf{y} \in R^{d}: d\left(\mathbf{g}^{(i)}(\mathbf{x}), \mathbf{g}^{(i)}(\mathbf{y})\right)<\varepsilon 0 \leqq i \leqq n\right\}
$$

needed to cover $\mathscr{D}$ and

$$
V\left(\mathbf{g}^{(n)}, \boldsymbol{\sigma} ; \varepsilon\right)=\sup _{\mathbf{x} \in \mathscr{L}} \ell\left(\mathbf{g}^{(n)} \boldsymbol{\sigma} \cap b\left(\mathbf{x}, \mathbf{g}^{(n)} ; \varepsilon\right)\right) .
$$

(d) There are 2 types of $\mathbf{g}$ 's that will result in different estimates for $\tau$ and $V$. They are those "near" $f$ and those "far away;" we distinguish between them through the use of the parameter $\beta$. For $\beta>0$, let

$$
\Omega_{\beta}=\left\{\mathbf{g} \in \Omega:\|\mathbf{f}-\mathbf{g}\|_{C^{k}}<\beta\right\} .
$$

A suitable choice for $\beta$ will be determined later.

Step 2. Estimation of $\tau$. The main point here is that sufficiently small noise does not increase the growth rate of distinct trajectories (and large noise is too rare to be of importance).

(a) Let $n_{0}$ be chosen so that

$$
\frac{1}{n_{0}} \ln N\left(\bigvee_{0}^{n_{0}-1} \mathbf{f}^{-l}\left(\alpha_{\varepsilon} \mid \mathscr{D}\right)\right)<h(\mathbf{f} \mid \mathscr{D})+2 \varepsilon_{0},
$$

and let $\mathscr{C}_{0}$ be a subcollection of $\bigvee_{0}^{n_{0}-1} \mathbf{f}^{-i}\left(\alpha_{\varepsilon}\right)$ that covers $\mathscr{D}$ and achieves the minimum cardinality. The number $n_{0}$ will be fixed throughout, and we will be thinking in terms of blocks of maps $\left\{\mathbf{g}_{1}, \ldots, \mathbf{g}_{n}\right\}$ of length $n_{0}$. A block is called a good block if $\mathbf{g}_{l} \in \Omega_{\beta} \forall i$; it is called a bad block if at least one $\mathbf{g}_{i} \notin \Omega_{\beta}$.

(b) If necessary we move the elements of $\alpha_{\varepsilon}$ slightly so that for any 2 elements $A$ and $B$ of $\bigvee_{0}^{n_{0}-1} \mathbf{f}^{-l}\left(\alpha_{\varepsilon}\right)$, either $A \cap B \neq \emptyset$ or their closures do not intersect (i.e., they do not touch along the boundary). We assume $\beta$ is sufficiently small so that if $\left\{\mathbf{g}_{1}, \ldots, \mathbf{g}_{n_{0}}\right\}$ is a good block, then there is a 1-1 correspondence between the elements of $\bigvee_{0}^{n_{0}-1} \mathbf{f}^{-l}\left(\alpha_{\varepsilon}\right)$ and those of $\bigvee_{0}^{n_{0}-1} \mathbf{g}^{-(l)}\left(\alpha_{\varepsilon}\right)$ and, moreover, the subcollection of $\bigvee_{0}^{n_{0}-1} \mathbf{g}^{-(l)}\left(\alpha_{\varepsilon}\right)$ corresponding to $\mathscr{C}_{0}$ covers $\mathscr{D}$.

(c) Let $\mathbf{g}_{0}, \mathbf{g}_{1}, \mathbf{g}_{2}, \ldots$ be given (and fixed for the rest of this step). For $j=1,2, \ldots$ we will pick a subcollection $\mathscr{C}$, of $\bigvee_{0}^{j n_{0}-1} \mathbf{g}^{-(l)}\left(\alpha_{\varepsilon}\right)$ which covers $\mathscr{D}$. The rules for constructing $\mathscr{C}_{j}$ from $\mathscr{C}_{j-1}$ are as follows: let $\left[A_{0}, \ldots, A_{(j-1) n_{0}-1}\right]$ represent an 
element of $\mathscr{C}_{j-1}$, i.e., $A_{i} \in \alpha_{\varepsilon}$ and $A_{0} \cap \mathbf{g}^{-(1)} A_{1} \cap \cdots \cap \mathbf{g}^{-\left((j-1) n_{0}-1\right)} A_{(J-1) n_{0}-1}$ is in $\mathscr{C}_{j-1}$. Then

Case 1. $\left\{\mathbf{g}_{(j-1) n_{0}}, \ldots, \mathbf{g}_{j n_{0}-1}\right\}$ is a good block and $A_{(j-1) n_{0}-1} \subset \mathscr{D}$. In this case we may attach any $\left[A_{(j-1) n_{0}}, \ldots, A_{j n_{0}-1}\right]$ in $\mathscr{C}_{0}$ to the given sequence and consider the resulting sequence as an element of $\mathscr{C}_{j}$. (Of course, many of these elements may be empty.) In this case the random maps are acting very much like $\mathbf{f}$.

Case 2. $\left\{\mathbf{g}_{(j-1) n_{0}}, \ldots, \mathbf{g}_{j n_{0}-1}\right\}$ is good and $A_{(j-1) n_{0}-1} \not \subset \mathscr{D}$ (i.e., previous noise has caused a drift out of $\mathscr{D})$. In this case we attach a sequence of the form $[A, \ldots, A]$, where $A \cap A_{(j-1) n_{0}-1} \neq \emptyset$. The number of such elements is $\leqq C_{d}$ (see Step 1(a)(i)), and since $\mathbf{f}=\mathbf{I d}$ outside of $\mathscr{D}$, we know from Step 1(a)(ii) that if $\beta$ is sufficiently small, then for every $\mathbf{x} \in A_{(j-1) n_{0}-1}, \exists A \in \alpha_{\varepsilon}$ such that $\mathbf{g}_{(j-1) n_{0}+i} \circ \cdots \circ \mathbf{g}_{(j-1) n_{0}} \mathbf{x} \in$ $A \forall i=0, \ldots, n_{0}-1$.

Case 3. $\left\{\mathbf{g}_{(j-1) n_{0}}, \ldots, \mathbf{g}_{j n_{0}-1}\right\}$ is a bad block (i.e., a large noise event occurs in this block). In this case we may attach sequences of the form $\left[B_{0}, \ldots, B_{n_{0}-1}\right]$ where $\mathbf{g}_{\left((j-1) n_{0}-1\right)} A_{(J-1) n_{0}-1} \cap B_{0} \neq \emptyset, \mathbf{g}_{\left((j-1) n_{0}\right)} B_{0} \cap B_{1} \neq \emptyset, \mathbf{g}_{\left((j-1) n_{0}+1\right)} B_{1} \cap B_{2} \neq \emptyset$, etc. The number of these new elements is difficult to control; however large noise events are rare.

Note that the $\mathscr{C}_{j}$ so obtained is a cover for $\mathscr{D}$, i.e., every possible trajectory has been accounted for.

(d) We view the sequence of maps $\mathbf{g}_{0}, \mathbf{g}_{1}, \ldots, \mathbf{g}_{j n_{0}-1}$ as a concatenation of $n_{0^{-}}$ blocks.

Claim 4. For some $C_{0}$ independent of $j$ we have for almost every sequence $\mathbf{g}_{1}, \mathbf{g}_{2}, \ldots$,

$$
\operatorname{card} \mathscr{C}_{j} \leqq e^{n_{0}\left(h+2 \varepsilon_{0}\right) \Gamma_{1}^{+}} e^{n_{0} C_{0} \Gamma_{1}^{-}}
$$

where $\Gamma_{j}^{+}$is the number of good blocks and $\Gamma_{j}^{-}$the number of bad blocks among the first $j$ blocks.

Proof. We proceed by induction, counting at the $j^{\text {th }}$ stage the maximum number of sequences that can be attached to each sequence in $\mathscr{C}_{j-1}$. In Case 1 , this number is $\leqq e^{n_{0}\left(h+2 \varepsilon_{0}\right)}$ by $2(\mathrm{a})$. In Case 2 , this number is $\leqq C_{d}$ (see Step 1(a)), which we may assume is $\leqq e^{n_{0}\left(h+2 \varepsilon_{0}\right)}$ if $n_{0}$ is chosen sufficiently large. In the last case, since there is a uniform Lipschitz constant $v_{\eta}$ a.s., we have that for every $A \in \alpha_{\varepsilon}, \mathbf{g}_{i} A$ is contained in a ball of fixed radius. By Step 1(a), this ball intersects $\leqq e^{C_{0}}$ elements of $\alpha_{\varepsilon}$ for some $C_{0}$.

We conclude Step 2 by noting that using Property 3 of Sect. $3, \tau_{j n_{0}}\left(\mathbf{g}^{\left(j n_{0}\right)}, \varepsilon\right) \leqq$ card $\mathscr{C}_{J} \leqq$ the number in Claim 4 .

\section{Step 3. Estimation of $V$.}

(a) The crucial observation is that Yomdin's proof (see Appendix A) works equally well for compositions of random maps, and that the estimates involved depend only on the $\ell^{\text {th }}$ derivatives, $1 \leqq \ell \leqq k$, of these maps. Let $\mathbf{g}_{0}, \mathbf{g}_{1}, \ldots$ be such that $\forall i,\left\|D^{l} \mathbf{g}_{i}\right\| \leqq C, 1 \leqq l \leqq k$ (condition 1 on $v_{\eta}$ ). Assuming the scaling 
condition in Step 1(b) and that $n_{0}$ is sufficiently large, we see from the argument in Appendix A that

$$
\frac{1}{j n_{0}} \ln V\left(\mathbf{g}^{\left(j n_{0}\right)}, \boldsymbol{\sigma} ; \varepsilon\right) \leqq \frac{1}{k j n_{0}} \ln \left\|D \mathbf{g}^{\left(j n_{0}\right)}\right\|+\varepsilon_{0}
$$

$\forall j \geqq$ some $j_{0}$.

(b) Claim 5.

$$
V\left(\mathbf{g}^{\left(j n_{0}\right)}, \boldsymbol{\sigma} ; \varepsilon\right) \leqq e^{n_{0} \Gamma_{j}^{+}\left(r(\mathbf{f}) / k+2 \varepsilon_{0}\right)} e^{n_{0} C_{1} \Gamma_{j}^{-}},
$$

where $C_{1}$ is a constant independent of $j$ and $\left\{\mathbf{g}_{i}\right\}$, and $\Gamma_{j}^{+}$and $\Gamma_{j}^{-}$are the number of good and bad blocks respectively among the first $j$ blocks of length $n_{0}$.

Proof. Write

$$
\frac{1}{j n_{0}} \ln \left\|D \mathbf{g}^{\left(j n_{0}\right)}\right\| \leqq \frac{1}{j} \sum_{l=1}^{j} \frac{1}{n_{0}} \ln \left\|D \mathbf{g}_{\left((l-1) n_{0}+1\right)}^{\left(i n_{0}\right)}\right\|,
$$

where $\mathbf{g}_{\left((i-1) n_{0}+1\right)}^{\left(i n_{0}\right)}$ denotes the composition $\mathbf{g}_{l n_{0}} \circ \cdots \circ \mathbf{g}_{(i-1) n_{0}+1}$. Assuming that $\beta$ is sufficiently small, we have $\left(1 / n_{0}\right) \ln \left\|D \mathbf{g}_{\left((j+1) n_{0}+1\right)}^{\left(j n_{0}\right)}\right\| \leqq\left(1 / n_{0}\right) \ln \left\|D \mathbf{f}^{n_{0}}\right\|+\varepsilon_{0} \leqq$ $r(\mathbf{f})+2 \varepsilon_{0}$ if $\left\{\mathbf{g}_{(t-1) n_{0}+1}, \ldots, \mathbf{g}_{n_{0}-1}\right\}$ is a good block. In general all we can say for a bad block is that $\left\|D \mathbf{g}_{\left((l-1) n_{0}\right)}^{\left(i n_{0}\right)}\right\| \leqq C_{1}^{n_{0}}$ for some $C_{1}$.

Step 4. The count. Summarizing, we have shown that for $v_{\eta}^{N}$-a.e. $\mathbf{g}_{1}, \mathbf{g}_{2}, \ldots$ and sufficiently small $\eta$, if we partition this sequence into blocks of length $n_{0}$, then the contribution to the $\tau$-term of the $j^{\text {th }}$ block is $\leqq e^{n_{0}\left(h+2 \varepsilon_{0}\right)}$ for a good block and $\leqq e^{C_{0} n_{0}}$ for a bad one. Also the $j^{\text {th }}$ block contributes $\leqq e^{n_{0}\left(r(\mathbf{f}) / k+2 \varepsilon_{0}\right)}$ to the $V$-term for a good block and $\leqq e^{C_{1} n_{0}}$ for a bad one. Since the blocks are i.i.d., we conclude that

$$
E\left(\ell\left(\mathbf{g}^{\left(j n_{0}\right)} \boldsymbol{\sigma}\right)\right) \leqq\left[P_{g} e^{n_{0}\left(h+r / k+4 \varepsilon_{0}\right)}+P_{b} e^{C_{2} n_{0}}\right]^{j},
$$

where $E\left(\ell\left(\mathbf{g}^{\left(j n_{0}\right)} \boldsymbol{\sigma}\right)\right)$ is the expected value of $\ell\left(\mathbf{g}^{\left(j n_{0}\right)} \boldsymbol{\sigma}\right), P_{g}$ is the probability of a good block, $P_{b}$ is the probability of a bad block, and $C_{2}=C_{0}+C_{1}$. This bound is clearly $\leqq e^{j n_{0}\left(h+r / k+5 \varepsilon_{0}\right)}$ if $P_{b}$ is smaller than some $\gamma$, and such a circumstance can be guaranteed by choosing $\eta$ small enough so that $1-\left(v_{\eta}\left(\Omega_{\beta}\right)\right)^{n_{0}} \leqq \gamma$.

\section{Upper Bound on the Growth Rate for Flows in the Limit $\boldsymbol{R}_{m} \rightarrow \infty$}

In this section we adapt the methods of Sect. 5 to flows, thereby completing the proof of Theorem 1. Consider again the magnetic induction equation

$$
\frac{\partial \mathbf{B}}{\partial t}=\mathbf{B} \cdot \nabla \mathbf{u}-\mathbf{u} \cdot \nabla \mathbf{B}+\frac{1}{R_{m}} \nabla^{2} \mathbf{B},
$$

where $\mathbf{u}$ is a $C^{k+1}, k \geqq 2$ divergence free vector field with compact support on $R^{d}$. For simplicity of notation write $\eta=1 / R_{m}$. In order to use the methods of Sect. 5, we need to produce a family of probability measures $\left\{v_{\eta}, \eta \geqq 0\right\}$ on $\Omega$, the space of $C^{k}$ volume preserving diffeomorphisms of $R^{d}$, so that $\left\{v_{\eta}\right\}$ satisfies the conditions at the beginning of Sect. 5 and also such that given $\mathbf{B}_{0}, \mathbf{B}_{n, \eta} \equiv \int D \mathbf{g}^{(n)} \mathbf{B}_{0} d v_{\eta}^{n}$ is the solution of (11) with initial conditions $\mathbf{B}_{0}$. 
We will describe two different ways of obtaining $\left\{v_{\eta}\right\}$, one using stochastic flows and the other a product formula for operators. Since the techniques involved are fairly standard, we will state the results here and outline the proofs in the appendices.

6.1. The Stochastic Differential Equations Approach. Let $\mathbf{u}$ be as above. (For technical reasons this method requires that $\mathbf{u}$ be of class $C^{k+1+\alpha}$ for $k \geqq 2, \alpha>0$.) Consider the SDE

$$
d \mathbf{X}_{t}=\mathbf{u} d t+\sqrt{2 \eta} d \mathbf{b}_{t},
$$

where $\mathbf{b}_{t}$ is standard Brownian motion on $R^{d}$. From the theory of stochastic flows (see e.g. Kunita (1990)) it follows that $\mathbf{X}(\mathbf{x}, t ; \omega)$ can be chosen in such a way that for each (fixed) $\omega, t \mapsto \mathbf{X}_{t, \omega}$ is a continuous path in the space of $C^{k}$ diffeomorphisms of $R^{d}$.

Lemma 1. Given an initial condition $\mathbf{B}_{0}$, define

$$
\mathbf{B}(\mathbf{x}, t)=E\left[D \mathbf{X}_{t, \omega}\left(\mathbf{X}_{t, \omega}^{-1}(\mathbf{x})\right) \mathbf{B}_{0}\left(\mathbf{X}_{t, \omega}^{-1}(\mathbf{x})\right)\right]
$$

Then $\mathbf{B}(\mathbf{x}, t)$ is the soultion of (11) with initial condition $\mathbf{B}_{0}$.

(Remember that $D(\cdot)$ here as elsewhere in this paper refers to spatial derivative, i.e., derivative with respect to $\mathbf{x}$.) See Appendix B for a proof.

Let $v_{\eta}$ be the distribution of $\mathbf{X}_{t=1, \omega}$. It follows from the Markovian property of the solution of (12) that the distribution of $\mathbf{X}_{n, \omega}$ is the same as that of $\mathbf{g}_{n} \circ \cdots \circ \mathbf{g}_{1}$, where the $\mathbf{g}_{i}$ 's are iid with law $v_{\eta}$. Condition 1 at the beginning of Sect. 5 follows from the fact that the Brownian term has constant coefficient. Condition 2 is a standard fact about stochastic flows (see e.g. Kunita (1990)). Theorem 1 now follows by the arguments of Sect. 5 .

6.2. The Product Formula Approach. We now consider the product formula construction. Let $N_{t}$ be the time $t$ propagator of the equation

$$
\frac{\partial \chi}{\partial t}=\chi \cdot \nabla \mathbf{u}-\mathbf{u} \cdot \nabla \chi
$$

(the Cauchy solution) and $M_{t}$ the time $t$ propagator of the diffusion equation

$$
\frac{\partial \chi}{\partial t}=\eta \nabla^{2} \chi
$$

We approximate the solution of (11) after time $t$ by the product formula $\mathbf{B}^{(\delta)}(\cdot, t)=$ $\left(M_{\delta} N_{\delta}\right)^{[t / \delta]} \mathbf{B}_{0}$, where $\mathbf{B}_{0}$ is the initial magnetic field and $\delta$ is the size of each time step. The convergence of this scheme, a standard result, is the subject of the following lemma:

Lemma 2. For fixed $t$ and $\eta$

$$
\lim _{\delta \rightarrow 0} \int_{R^{d}}\left|\mathbf{B}(\mathbf{x}, t)-\mathbf{B}^{(\delta)}(\mathbf{x}, t)\right| d \mathbf{x}=0,
$$

where $\mathbf{B}$ is the solution to Eq. (11).

The proof is sketched in Appendix C. 
Let us now return to calculating with maps. For fixed $\delta=1 / m, m$ a large integer, let $v_{\eta, \delta}$ be the distribution of

$$
\mathbf{g}=\left(\phi_{\delta}+\omega_{m}(\delta)\right) \circ \cdots \circ\left(\phi_{\delta}+\omega_{1}(\delta)\right),
$$

where $\phi_{s}$ is the flow generated by $\mathbf{u}$ and the $\omega_{i}(\delta)$ are $d$-dimensional random vectors with i.i.d. Gaussian entries of mean 0 and variance $2 \eta \delta$. An easy calculation shows that

$$
\mathbf{B}^{(\delta)}(\cdot, n)=\int_{\Omega^{n}}\left(D \mathbf{g}^{(n)} \mathbf{B}_{0}\right) v_{\eta, \delta}^{n}\left(d \mathbf{g}_{1}, \ldots, d \mathbf{g}_{n}\right)
$$

We need the following lemma controlling the uniform dependence on $\eta$ of $v_{\eta, \delta}$ as $\delta \rightarrow 0$ to finish the proof:

Lemma 3. For given $\varepsilon>0$

$$
\lim _{\eta \rightarrow 0} \lim _{\delta \rightarrow 0} v_{\eta, \delta}\left\{\mathbf{g}:\left\|\mathbf{g}-\phi_{1}\right\|_{C^{k}}<\varepsilon\right\}=1 .
$$

This statement takes the place of the previous Condition 2. The proof can be found in Appendix C.

We further make the following claim:

Claim 6. Given $\varepsilon_{0}>0, \exists \eta_{0}>0$ such that for any fixed $\eta<\eta_{0}, \exists N_{\eta} \in Z^{+}$and $\delta_{\eta}>0$ such that $\forall n \geqq N_{\eta}, \delta \leqq \delta_{\eta}$, if $\boldsymbol{\sigma}:[0,1] \rightarrow \mathscr{D}$ is a $C^{k}$ curve with $\|\boldsymbol{\sigma}\|_{k} \leqq 1$, then

$$
\int \ell\left(\mathbf{g}^{(n)} \boldsymbol{\sigma}\right) v_{\eta, \delta}^{n}\left(d \mathbf{g}^{n}\right) \leqq e^{n\left(h(\mathbf{f})+r(\mathbf{f}) / k+5 \varepsilon_{0}\right)} .
$$

The proof is identical to that for maps provided $\eta_{0}$ is chosen small enough to assure that the probability of occurrence of a "bad block" is sufficiently small. The existence of such an $\eta_{0}$ is guaranteed by Lemma 3 .

To complete the proof of Theorem 1 , we first replace a given initial condition $\mathbf{B}_{0}$ by $\tilde{\mathbf{B}}_{0}$ as in Sect. 4. Then we argue as in the paragraphs preceding Claim 3 in Sect. 5 that for arbitrary $\eta, \delta$, and $n$,

$$
\int_{R^{d}}\left|\mathbf{B}^{(\delta)}(\mathbf{x}, n)\right| d \mathbf{x} \leqq C \sup _{\boldsymbol{\sigma}} \int_{\Omega^{n}} \ell\left(\mathbf{g}^{n} \boldsymbol{\sigma}\right) d v_{\eta, \delta}^{n},
$$

where $C$ depends on $\mathbf{B}_{0}$ but not on $\eta, \delta$ or $n$. Let $\varepsilon_{0}>0$ be given and let $\eta<\eta_{0}$ be as in Claim 6. We now estimate the growth rate of $\int|\mathbf{B}(\mathbf{x}, n)| d \mathbf{x}$ as $n \rightarrow \infty$ for this fixed $\eta$. Temporarily fix $n \geqq N_{\eta}$ where $N_{\eta}$ is as in Claim 6 . We choose $\delta<\delta_{\eta}$ such that the difference in Lemma 2 is less than 1. (Note that $\delta$ must be allowed to depend on $n$ since there is no uniformity in this approximation as time tends to $\infty$.) It then follows from Lemma 2 , our estimate on $\int\left|\mathbf{B}^{(\delta)}(\mathbf{x}, n)\right| d \mathbf{x}$ above, and Claim 6, that

$$
\int_{R^{d}}|\mathbf{B}(\mathbf{x}, n)| d \mathbf{x} \leqq 1+C e^{n\left(h+r / k+5 \varepsilon_{0}\right)} .
$$

\section{Further Remarks}

The range of applicability of Theorems 1 and $1^{\prime}$ can be extended in several important ways. We note first that while we have been assuming that the map $f$ or flow $\mathbf{u}$ is deterministic, the results also hold under mild restrictions for random 
maps and flows such as the "renovating flow" dynamos (Zel'dovich et al. (1988), Gilbert and Bayly (1992)). The extension to random systems of the key steps of the $R_{m}=\infty$ results, namely definitions of topological entropy and line stretching and the Yomdin upper bound, can be found in Kifer (1986) and Kifer and Yomdin (1988). The arguments contained in this paper also extend to random distributions in a straightforward manner. Furthermore the bound of Theorem 1 applies for any $R_{m}$ with $h(\mathbf{f})$ replaced by $h(\mathbf{g})$, where the distribution of $\mathbf{g}$ consists of Gaussian perturbations of $\mathbf{f}$ with variance $2 R_{m}^{-1}$. Secondly the proof is easily modified to apply to periodic boundary conditions - the main difference is that Case 2 of Step 2(c) in Sect. 5 is no longer necessary. Furthermore for periodic boundary conditions $\mathbf{B}_{0}$ need only be continuous; diffusion will smooth the field after an arbitrarily small time. (The same argument cannot be used for the original case because of apparent technical difficulties arising from unboundedness of the domain). We note also that $\mathbf{u}$ may be time periodic. A periodic flow in $R^{d}$ can be embedded as a steady flow in $R^{d} \times S \subset R^{d+1}$. In this case diffusion acts only in the $R^{d}$ directions. In principle bounds involving topological entropy can also be given for more general time-dependent flows.

Certain restrictions of Theorems 1 and $1^{\prime}$ should be noted as well. As has already been seen, for technical reasons we assume the initial field is contained in a bounded region. In addition we assume that the diffusivity is constant in space. From the point of view of this paper, a non-constant diffusivity would result in spatially varying noise. Thus diffusion could stretch the magnetic field and in principle have a direct role in the dynamo process. For the same reason issues concerned with fast dynamo action surrounded by an insulating region (Hollerbach et al. (1995)) are not addressed here.

The techniques of this paper can also be applied to the evolution of a passive scalar gradient field. The equation for the evolution of a passive scalar quantity $\theta$ is

$$
\frac{\partial \theta}{\partial t}+\mathbf{u} \cdot \nabla \theta=\frac{1}{P e} \nabla^{2} \theta
$$

where the Peclet number $P e$ is a dimensionless parameter measuring the relative strengths of advective and diffusive processes. Taking the gradient of (13) we get

$$
\frac{\partial}{\partial t}(\nabla \theta)+\mathbf{u} \cdot \nabla(\nabla \theta)=-(\nabla \mathbf{u})^{T} \nabla \theta+\frac{1}{P e} \nabla^{2}(\nabla \theta)
$$

where $(\nabla \mathbf{u})^{T}$ is the transpose of $\nabla \mathbf{u}$. When $P e=\infty$ this equation is identical in form to the time evolution equation for a field of material area elements. To see this, let $\mathbf{b} \times \mathbf{c}$ be a material area element. Abbreviating $d / d t=\partial / \partial t+\mathbf{u} \cdot \nabla$, by volume preservation we have for any material vector $\mathbf{a}$,

$$
0=\frac{d}{d t}(\mathbf{a} \cdot(\mathbf{b} \times \mathbf{c}))=\mathbf{a} \cdot\left[(\nabla \mathbf{u})^{T}(\mathbf{b} \times \mathbf{c})+\frac{d}{d t}(\mathbf{b} \times \mathbf{c})\right]
$$

Since a was arbitrary we obtain

$$
\frac{d}{d t}(\mathbf{b} \times \mathbf{c})=-(\nabla \mathbf{u})^{T}(\mathbf{b} \times \mathbf{c})
$$


As a Yomdin inequality holds for area elements as well as line elements, it can be shown that at $P e=\infty$,

$$
\lim _{n \rightarrow \infty} \frac{1}{n} \ln \int_{\mathscr{D}}|\nabla \theta| d \mathbf{x} \leqq h(\mathbf{f})+\frac{2 r(\mathbf{f})}{k},
$$

where, as before, $\mathbf{f}$ is a $C^{k}$ map (or time 1 integration of a steady or period 1 flow). This result might be interpreted as requiring $h(\mathbf{f})+2 r(\mathbf{f}) / k>0$ as a necessary (although not sufficient) condition for efficient mixing at large Peclet number. If $k=\infty$ then in fact chaos becomes a necessary condition for efficient mixing.

\section{Proof of the Lower Bound}

The proof of Theorem 2 uses some basic techniques from smooth ergodic theory. These techniques are standard to workers in the subject, but without a substantial amount of notation and preliminaries it is difficult to make precise statements. Instead of giving a formal proof we will elucidate the main geometric ideas, referring the reader interested in technical details to an expository article (Young (1995)) or Pesin's original paper (Pesin (1978)). An argument similar to ours has been used by Newhouse (1988). Throughout this section let $\mathbf{f}$ be as in Theorem 2 .

\section{Idea 1. Measures maximizing entropy}

If $\mu$ is an $\mathbf{f}$-invariant Borel probability measure on $\mathscr{D}$, let $h_{\mu}(\mathbf{f})$ denote the metric entropy of $\mathbf{f}$ with respect to $\mu$ (see Walters (1982) for a definition). In much the same way that topological entropy measures chaos in a topological sense, metric entropy measures randomness in the sense of probability. The following variational principle is well known:

$$
h(\mathbf{f})=\sup _{\mu} h_{\mu}(\mathbf{f}) .
$$

The supremum is taken over all f-invariant probability measures; it is also adequate to only consider ergodic measures.

Our first step is to pick (and fix throughout) an ergodic measure $\mu$ with $h_{\mu}(\mathbf{f})>$ $h(\mathbf{f})-\varepsilon_{0}$. More likely than not, $\mu$ is a singular measure, i.e., it lives on a set of 0 volume. The purpose of this proof is to show that points that are typical with respect to $\mu$, exceptional as they may be from the point of view of volume, may have a significant influence on the growth rate of $\int\left|\mathbf{B}_{n}\right|^{q} d \mathbf{x}$.

\section{Idea 2. Lyapunov exponents and special coordinates}

According to a theorem of Oseledets (1968), there exists a set of numbers $\lambda_{1}$ $>\cdots>\lambda_{r}$ with multiplicities $m_{1}, \ldots, m_{r}$ respectively such that at $\mu$-a.e. $\mathbf{x}$ the tangent space $T_{\mathbf{x}} \mathscr{D}$ splits into $T_{\mathbf{x}} \mathscr{D}=E_{1}(\mathbf{x}) \oplus \cdots \oplus E_{r}(\mathbf{x})$ in such a way that $\forall \mathbf{v} \in E_{i}(\mathbf{x})$

$$
\lim _{n \rightarrow \infty} \frac{1}{n} \ln \left|D \mathbf{f}_{\mathbf{x}}^{n} \mathbf{v}\right|=\lambda_{i} .
$$


These numbers are called the Lyapunov exponents of $(\mathbf{f}, \mu)$. The subspaces $E_{i}(\mathbf{x})$ vary measurably with $\mathbf{x}$-they vary continuously if we are willing to disregard a set of arbitrarily small measure.

In general Lyapunov exponents are asymptotic growth rates, but there are point dependent changes of coordinates due to Pesin (1978) that allow us to see f locally as small perturbations of linear maps with eigenvalues $\exp \left(\lambda_{i}\right)$. More precisely, at $\mu$-a.e. $\mathbf{x}$ there exists a differentiable change of coordinates $\boldsymbol{\Phi}_{\mathbf{x}}: N_{\mathbf{x}} \rightarrow U_{\mathbf{x}}$, where $N_{x}$ is a small neighborhood of $\mathbf{0}$ in $R^{d}$ and $U_{\mathbf{x}}$ is a small neighborhood of $\mathbf{x}$, with the properties that $E_{r}(\mathbf{x})$;

1. $\boldsymbol{\Phi}_{\mathbf{x}}(\mathbf{0})=\mathbf{x}$ and $D \boldsymbol{\Phi}_{\mathbf{x}}(\mathbf{0})$ carries the splitting $R^{m_{1}} \oplus \cdots \oplus R^{m_{\imath}}$ to $E_{1}(\mathbf{x}) \oplus \cdots \oplus$

2. if $\tilde{\mathbf{f}}_{\mathbf{x}}: N_{\mathbf{x}}: \rightarrow N_{\mathbf{f}}$ is the representation of $\mathbf{f}$ in the new coordinates, then $\tilde{\mathbf{f}}_{\mathbf{x}}$ is $C^{1}$ near $D \tilde{\mathbf{f}}_{\mathbf{x}}(\mathbf{0})$ and $D \tilde{\mathbf{f}}_{\mathbf{x}}(\mathbf{0})$ is a linear map with $\left|D \tilde{\mathbf{f}}_{\mathbf{x}}(\mathbf{0}) \mathbf{v}\right| \sim \exp \left(\lambda_{l} \pm \varepsilon\right)|\mathbf{v}| \forall \mathbf{v} \in R^{m_{l}}$.

It should be noted that these change of coordinates distort distances by arbitrarily large amounts (depending on $\mathbf{x}$ ) and that there are various technical problems of which one ought to be aware when working with them (see e.g. Young (1995)).

\section{Idea 3. The influence of $\mu$-typical points on dynamo growth}

We will assume in this paragraph that we are working in our special coordinates so that locally $\mathbf{f}$ resembles a linear map with multipliers $\exp \left(\lambda_{l}\right)$ of multiplicity $m_{l}$. In fact for a typical $\mathbf{x}$ let us think of $U_{\mathbf{x}}$ as a product of the form $U_{\mathbf{x}}^{u} \times U_{\mathbf{x}}^{s}$, where $U_{\mathbf{x}}^{u}$ is a disk contained in $\oplus E_{l}(\mathbf{x}), \lambda_{l} \geqq 0$, and $U_{\mathbf{x}}^{s}$ is a disk contained in $\oplus E_{i}(\mathbf{x})$, $\lambda_{i}<0$. As before let $b(\mathbf{y}, \varepsilon ; n)=\left\{\mathbf{z}: d\left(\mathbf{f}^{l} \mathbf{y}, \mathbf{f}^{i} \mathbf{z}\right)<\varepsilon \forall 0 \leqq i<n\right\}$. Consider $\mathbf{y}$ very near the center of $U_{\mathbf{x}}$ and assume that diam $U_{\mathbf{x}} \approx \varepsilon$. Then $b(\mathbf{y}, \varepsilon ; n) \approx C \times U_{\mathbf{x}}^{s}$ for some $C$ with cross-sectional area $\sim \exp \left(-n \sum \lambda_{i}^{+} m_{i}\right), \lambda_{i}^{+} \equiv \max \left(\lambda_{i}, 0\right)$. (This is true exactly if $\mathbf{f}$ is locally linear; in our coordinates the nonlinearities are mild.)

Next let $h=h_{\mu}(\mathbf{f})$. A property of metric entropy is that given any set $U$ of positive measure, for $n$ sufficiently large there exists $O(\exp (n h))$ points in $U$ that are $(n, \varepsilon)$-separated, i.e., $\exists \mathbf{x}_{1}, \ldots, \mathbf{x}_{k} \in U, k \sim \exp (n h)$, such that $b\left(\mathbf{x}_{i}, \varepsilon ; n\right) \cap$ $b\left(\mathbf{x}_{j}, \varepsilon ; n\right)=\emptyset$ whenever $i \neq j$. We pick these points in $U_{\mathbf{x}}$ very near $\mathbf{x}$ and let $A_{n}=\bigcup_{l} b\left(\mathbf{x}_{i}, \varepsilon ; n\right)$.

To complete the proof we now exhibit a vector field $\mathbf{B}_{0}$ with the desired growth rate. All that we really require of $\mathbf{B}_{0}$ is that in $U_{\mathbf{x}}$ it points roughly in the direction of $E_{1}(\mathbf{x})$. Then if $q=\operatorname{dim} U_{\mathbf{x}}^{u}$, we will have

$$
\begin{aligned}
\int_{A_{n}}\left|D \mathbf{f}^{n} \mathbf{B}_{0}\right|^{q} d \mathbf{x} & \sim e^{q \lambda_{1} n} \cdot\left(\text { Lebesgue measure of } A_{n}\right) \\
& \sim e^{q \lambda_{1} n} \cdot e^{-\left(\sum \lambda_{l}^{+} m_{i}\right) n} e^{n h} \geqq e^{n h},
\end{aligned}
$$

and so

$$
\liminf \frac{1}{n} \ln \int_{\mathscr{Q}}\left|\mathbf{B}_{n}\right|^{q} d \mathbf{x} \geqq h .
$$

If $h=0$ there is nothing to prove. If $h>0$ then $h \leqq \sum \lambda_{i}^{+} m_{i}$ by Ruelle's inequality (1978) so $\lambda_{1}>0$. The same reasoning tells us that $(\mathbf{f}, \mu)$ must have a strictly negative exponent; hence any $q \geqq d-1$ will work in (14). If $\mathbf{f}$ is the time 1 map of a flow then in addition to the negative exponent there is a zero exponent and so $q \geqq d-2$ suffices. 


\section{Appendices}

\section{A. Outline of the Proof of Yomdin's Theorem}

We present here a proof of Claim 2 following Yomdin (1987). The proof uses the following approximation lemma which allows one to renormalize after each iteration:

Lemma 4. Let $b(\mathbf{x} ; 1)$ and $b(\mathbf{x} ; 2)$ be the balls of radius 1 and 2 around a point $\mathbf{x}$ in $R^{d}$. Let $\mathbf{f}: b(\mathbf{x}, 2) \rightarrow R^{d}$ be a $C^{k}$ map with $\left\|D^{s} \mathbf{f}\right\| \leqq M, 1 \leqq s \leqq k$, and let $\boldsymbol{\sigma}:[0,1] \rightarrow b(\mathbf{x} ; 2)$ be a $C^{k}$ curve with $\|\boldsymbol{\sigma}\|_{k} \leqq 1$. Then there exist a constant $\kappa$ of the form $\kappa=\mu M^{1 / k}$ (with $\mu$ depending only on $k$ and $d$ ) disjoint intervals $I_{1}, \ldots, I_{\kappa} \subset[0,1]$ such that

1.

$$
(\mathbf{f} \circ \boldsymbol{\sigma})^{-1} b(\mathbf{f}(\mathbf{x}) ; 1) \subset \bigcup_{j} I_{j} \subset(\mathbf{f} \circ \boldsymbol{\sigma})^{-1} b(\mathbf{f}(\mathbf{x}) ; 2),
$$

2. If $\psi_{j}$ is an affine contraction mapping $[0,1]$ onto $I_{j}$, then

$$
\left\|D^{s}\left(\mathbf{f} \circ \boldsymbol{\sigma} \circ \psi_{j}\right)\right\| \leqq 1, \quad 1 \leqq s \leqq k
$$

Lemma 4 deals with the approximation of $C^{k}$ maps by polynomials and has nothing to do with dynamics. We will omit its proof.

Consider now $\mathbf{f}$ as in Theorems 1 or $1^{\prime}$. We define a map $\hat{\mathbf{f}}$ by blowing up $\mathbf{f}$ to $\hat{\mathbf{f}}=(1 / \varepsilon) \mathbf{f}(\varepsilon \mathbf{x})$. For $\varepsilon$ sufficiently small,

$$
\max _{s=1, . ., k}\left\|D^{s} \hat{\mathbf{f}}\right\|=\|D \hat{\mathbf{f}}\|=\|D \mathbf{f}\|
$$

Choose $\varepsilon$ such that $(15)$ holds. Given a $C^{k}$ curve $\sigma$ define a $C^{k}$ curve $\hat{\sigma}$ by blowing up $\boldsymbol{\sigma}$ to $\hat{\boldsymbol{\sigma}}=(1 / \varepsilon) \boldsymbol{\sigma}$ and assume for the moment that $\|\hat{\boldsymbol{\sigma}}\|_{k} \leqq 1$. We now apply the lemma to $\hat{\mathbf{f}}$ and $\hat{\boldsymbol{\sigma}}$ to get the maps $\psi_{j}$ and thus see that for any $\mathbf{x}$,

$$
\ell(\hat{\mathbf{f}} \hat{\boldsymbol{\sigma}} \cap \hat{b}(\mathbf{x}, 1 ; 1)) \leqq \kappa
$$

where $\kappa=\mu(k, d)\|D \mathbf{f}\|^{1 / k}$. We now repeat this process again and again, defining a new curve $\hat{\boldsymbol{\sigma}}^{\prime}$ from the previous curve $\hat{\boldsymbol{\sigma}}$ by $\hat{\boldsymbol{\sigma}}^{\prime}=\hat{\mathbf{f}} \circ \hat{\boldsymbol{\sigma}} \circ \psi_{j}$ (summing over all the $\psi_{J}$ 's). Then for any $\mathbf{x}$,

$$
\ell\left(\hat{\mathbf{f}}^{n} \hat{\boldsymbol{\sigma}} \cap \hat{b}(\mathbf{x}, n ; 1)\right) \leqq \kappa^{n}
$$

This implies that

$$
\limsup _{n \rightarrow \infty} \frac{1}{n} \ln \left[\ell\left(\mathbf{f}^{n} \boldsymbol{\sigma} \cap b(\mathbf{x}, n ; \varepsilon)\right)\right] \leqq \ln \mu(k)+\frac{1}{k} \ln \|D \mathbf{f}\|,
$$

which is in the direction of the desired result.

We remark first that in the process of blowing up $\boldsymbol{\sigma},\left\|D^{s} \hat{\boldsymbol{\sigma}}\right\|_{k}$ increases for $s>1$. We may however subdivide $\hat{\boldsymbol{\sigma}}$ into shorter curves $\hat{\boldsymbol{\sigma}}_{J}$ in such a way that, after reparametrizing, each $\hat{\boldsymbol{\sigma}}_{j}$ satisfies $\left\|\hat{\boldsymbol{\sigma}}_{j}\right\|_{k} \leqq 1$. The number of subdivisions depends only on $k$ and $\varepsilon$ (and not on $\boldsymbol{\sigma}$ ). 
To go from (16) to the desired result, we use the standard trick of working with a power of $\mathbf{f}$. Let $\varepsilon_{0}$ be given. Select $\varepsilon>0$ and an integer $p$ so that

1. $(1 / p) \ln \mu(k)<(1 / 3) \varepsilon_{0}$.

2. $\left(1 / k p^{\prime}\right) \ln \left\|D \mathbf{f}^{p^{\prime}}\right\|<r(\mathbf{f})+(1 / 3) \varepsilon_{0}$ for $p^{\prime} \geqq p$.

3. $\hat{\mathbf{f}}^{p}=\mathbf{f}^{p}$ blown up by $1 / \varepsilon$ has property (15).

4. $(1 / p) h\left(\mathbf{f}^{p} ; \varepsilon\right)<h(\mathbf{f})+\left(\varepsilon_{0} / 3\right)$. Here the $\varepsilon$ in $h\left(\mathbf{f}^{p} ; \varepsilon\right)$ refers to the $\varepsilon$ in definition $(8)$.

Then for $n \geqq 1$ (with $b(\mathbf{x}, n ; \varepsilon)$ calculated using the map $\left.\mathbf{f}^{p}\right)$,

$$
\begin{aligned}
\limsup _{n \rightarrow \infty} \frac{1}{n p} \ln \left(\mathbf{f}^{n p} \boldsymbol{\sigma}\right) & =\frac{1}{p} \limsup _{n \rightarrow \infty} \frac{1}{n} \ln \ell\left(\mathbf{f}^{n p} \boldsymbol{\sigma}\right) \\
& \leqq \frac{1}{p} h\left(\mathbf{f}^{p} ; \varepsilon\right)+\frac{1}{p} \limsup _{n \rightarrow \infty} \frac{1}{n} \ln \left[\ell\left(\left(\mathbf{f}^{p}\right)^{n} \boldsymbol{\sigma} \cap b(\mathbf{x}, n ; \varepsilon)\right)\right] \\
& \leqq h(\mathbf{f})+\frac{r(\mathbf{f})}{k}+\varepsilon_{0}
\end{aligned}
$$

independent of choice of $\boldsymbol{\sigma}$, as was to be shown.

\section{B. Details on Stochastic Flows}

Proof of Lemma 1. Since $\mathbf{X}_{t, \omega}$ is in fact $C^{3}, \mathbf{B}(\mathbf{x}, t)$ as defined in Lemma 1 is at least $C^{2}$ in $\mathbf{x}$ so the right side of $(11)$ is well-defined. For $\left(\mathbf{x}_{0}, t_{0}\right) \in R^{d} \times[0, \infty)$ let

$$
\frac{\partial \mathbf{B}}{\partial t}\left(\mathbf{x}_{0}, t_{0}\right)=\lim _{t \rightarrow 0^{+}} \frac{\mathbf{B}\left(\mathbf{x}_{0}, t_{0}+t\right)-\mathbf{B}\left(\mathbf{x}_{0}, t_{0}\right)}{t} .
$$

We will show that $\forall\left(\mathbf{x}_{0}, t_{0}\right)$ this limit exists and is equal to the right side of (11). (To conclude that $\partial \mathbf{B} / \partial t$ really exists we will observe that as $t \rightarrow 0^{+}$the convergence is in fact uniform in $t_{0}$ for $t_{0}$ in any finite time interval. This fact together with the continuity of $t_{0} \mapsto \partial \mathbf{B} / \partial t\left(\mathbf{x}_{0}, t_{0}\right)$ proves that $\partial \mathbf{B} / \partial t^{+}=\partial \mathbf{B} / \partial t^{-} \forall t_{0}>0$.)

To study $\partial \mathbf{B} / \partial t^{+}$, consider the backward derivative process associated with (12). More precisely, consider

$$
\begin{cases}d \mathbf{Y}_{t}=-\mathbf{u} d t+\sqrt{2 \eta} d \mathbf{b}_{t}, & \mathbf{Y}(0)=\mathbf{x}_{0} \\ d \mathbf{J}_{t}=-D \mathbf{u}, & \mathbf{J}(0)=\mathbf{I d}\end{cases}
$$

on $R^{d} \times G L(R, d)$, and introduce the function

$$
\mathbf{f}(\mathbf{Y}, \mathbf{J})=\mathbf{J}^{-1} \mathbf{B}\left(\mathbf{Y}, t_{0}\right) .
$$

Then using the fact that $\mathbf{X}_{t}^{-1}$ and $\mathbf{Y}_{t}$ have the same distributions, we have for $t>0$,

$$
\mathbf{B}\left(\mathbf{x}_{0}, t_{0}+t\right)=E\left[\left(D \mathbf{X}_{t, \omega}\right) \mathbf{x}_{-t, \omega}\left(\mathbf{x}_{0}\right) \mathbf{B}\left(\mathbf{X}_{-t, \omega}\left(\mathbf{x}_{0}\right), t_{0}\right)\right]=E(\mathbf{f}(\mathbf{Y}(t), \mathbf{J}(t))),
$$

so that

$$
\frac{\partial \mathbf{B}}{\partial t^{+}}=\lim _{t \rightarrow 0^{+}} \frac{1}{t} E\left[\mathbf{f}(\mathbf{Y}(t), \mathbf{J}(t))-\mathbf{f}\left(\mathbf{x}_{0}, \mathbf{I d}\right)\right]
$$


Note that $\mathbf{f}$ is $C^{2}$. Ito's formula tells us that

$$
\begin{aligned}
\mathbf{f}(\mathbf{Y}(t), \mathbf{J}(t))-\mathbf{f}\left(\mathbf{x}_{0}, \mathbf{I d}\right)= & \eta \int_{0}^{t} \frac{\partial^{2} \mathbf{f}}{\partial \mathbf{Y}^{2}} d s+\int_{0}^{t}\left[\left(\frac{\partial \mathbf{f}}{\partial \mathbf{Y}}, \frac{\partial \mathbf{f}}{\partial \mathbf{J}}\right) \cdot\left(\begin{array}{c}
-\mathbf{u} \\
-D \mathbf{u}
\end{array}\right)\right] d s \\
& +\sqrt{2 \eta} \int_{0}^{t} \frac{\partial \mathbf{f}}{\partial \mathbf{Y}} d \mathbf{b}_{s}
\end{aligned}
$$

Let us call the three terms in this equation (i), (ii), and (iii). Then $E$ (iii) $=0$. since (i) and (ii) are ordinary integrals, one verifies (using the uniform boundedness of $\mathbf{B}\left(\mathbf{x}, t_{0}\right), \mathbf{u}$, and $D \mathbf{u}$, and standard large deviation estimates for the trajectories of $(\mathbf{Y}(t), \mathbf{J}(t)))$ that

$$
\lim _{t \rightarrow 0^{+}} \frac{1}{t} E(i)=\eta\left(\nabla^{2} \mathbf{B}\right)\left(\mathbf{x}_{0}, t_{0}\right)
$$

and

$$
\lim _{t \rightarrow 0^{+}} \frac{1}{t} E(i i)=(\mathbf{B} \cdot \nabla \mathbf{u}-\nabla \mathbf{B} \cdot \mathbf{u})\left(\mathbf{x}_{0}, t_{0}\right)
$$

\section{Details on Operator Splitting}

Proof of Lemma 2. This is a standard and well-known result of operator splitting arising in slightly different forms in many different contexts. For completeness we outline here a proof using a particularly simple argument found in Chorin et al. (1978) p. 209.

Define the operator $K_{t}=M_{t} \circ N_{t}$, where $N_{t}$ is the time $t$ propagator of the evolution equation

$$
\frac{\partial}{\partial t} \boldsymbol{\chi}=\chi \cdot \nabla \mathbf{u}-\mathbf{u} \cdot \nabla \boldsymbol{\chi}
$$

(we assume $\mathbf{u}$ is $C^{k+1}, k \geqq 2$ ) and $M_{t}$ is the time $t$ propagator of the evolution equation

$$
\frac{\partial}{\partial t} \chi=\eta \nabla^{2} \chi
$$

Let $F_{t}$ be the time $t$ propagator for

$$
\frac{\partial}{\partial t} \chi=\chi \cdot \nabla \mathbf{u}-\mathbf{u} \cdot \nabla \chi+\eta \nabla^{2} \chi
$$

The object is to show that in the limit $\delta \rightarrow 0, K_{\delta}^{[t / \delta]}$ converges to $F_{t}$ for any $t$. Two basic properties are necessary to demonstrate convergence. First consistency requires that

$$
\left.\frac{d}{d t} K_{t}\right|_{t=0}=\left.\frac{d}{d t} F_{t}\right|_{t=0}
$$

Consistency follows immediately from the limit

$$
\frac{1}{t}\left(K_{t} \chi-\chi\right)=\frac{1}{t} M_{t}\left(N_{t} \chi-\chi\right)+\frac{1}{t}\left(M_{t} \chi-\chi\right) \rightarrow\left(\left.\frac{d}{d t} N_{t}\right|_{t=0}+\left.\frac{d}{d t} M_{t}\right|_{t=0}\right) \chi
$$


as $t \rightarrow 0$. The second property required, stability, is boundedness of $K_{\delta}^{[t / \delta]}$ as $\delta \rightarrow 0$ for small $t$. Stability can be shown, for example, by expanding the Green's function for $K_{\partial}^{[t / \delta]}$ for small $t$. We will not carry out the expansion here although, in fact, this argument can be found as part of the proof of Lemma 3.

Using consistency and stability it is then easily shown that $\left(F_{t}-K_{\delta}^{[t / \delta]}\right) \chi$ converges to 0 for small $t$. The convergence for arbitrary $T$ then follows from a compactness argument.

Proof of Lemma 3. We will show for sufficiently small $\eta$ and large $m$ that the two equations

$$
\begin{aligned}
\dot{\mathbf{x}} & =\mathbf{u}, \quad \mathbf{x}(0)=\mathbf{x}_{0}, \\
\dot{\mathbf{x}}_{\eta} & =\mathbf{u}+\sum_{j=1}^{m} \mathbf{a}_{j} \delta(t-j / m), \quad \mathbf{x}_{\eta}(0)=\mathbf{x}_{0},
\end{aligned}
$$

where $\mathbf{a}_{j}$ are iid $d$-dimensional Gaussian random variables with mean 0 and variance $2 \eta / m$, have solutions that remain uniformly close pointwise in $\mathbf{x}_{0}$ as $m \rightarrow \infty$ with probability approaching 1 as $\eta \rightarrow 0$. The higher derivatives are left to the reader.

First we consider the linearization of $\dot{\mathbf{x}}_{\eta}-\dot{\mathbf{x}}$ around the trajectory $\mathbf{x}(t), \mathbf{x}(0)=$ $\mathbf{x}_{0}$. That is, we consider the equation

$$
\dot{\boldsymbol{\xi}}=(\nabla \mathbf{u}) \boldsymbol{\xi}+\sum_{j=1}^{m} \mathbf{a}_{j} \delta(t-j / m) .
$$

The first task is to estimate the first passage time for $|\xi|$, i.e., to estimate the probability that $|\xi| \geqq c$ occurs first at time $t$. Now Eq. (18) can be integrated to yield the solution

$$
\boldsymbol{\xi}(t)=\sum_{j=1}^{[t m]} \mathbf{J}(\mathbf{x}(j / m)+\boldsymbol{\xi}(j / m), t-j / m) \mathbf{a}_{j},
$$

(showing that $|\xi|$ is uniformly bounded with high probability). Let $\alpha=\sup \|\nabla \mathbf{u}\|$. Then $\|\mathbf{J}(\mathbf{x}(j / m)+\xi(j / m), t-j / m)\| \leqq e^{\alpha}$. The probability of first passage of the sum (19) of Gaussian random variables is less than or equal to the first passage probability of the sum of Gaussian random variables

$$
\xi^{\prime}(t)=e^{\alpha} \sum_{j=1}^{[t m]} \mathbf{a}_{j}
$$

In the limit of $m \rightarrow \infty$ the probability $f(z, t)$ that $\left|\xi^{\prime}\right|$ exceeds $z>0$ is given by (Feller (1957))

$$
f(z, t)=\frac{z e^{\alpha}}{\sqrt{\pi \eta t^{3}}} \varepsilon^{-\left(z e^{\alpha}\right)^{2} / 4 \eta t}
$$

In particular for $\varepsilon>0$

$$
f\left(\eta^{1 / 2-\varepsilon}, 1\right)=\frac{2 \beta}{\sqrt{\pi}} e^{-\beta^{2}}
$$

where $\beta=e^{\alpha} \eta^{-\varepsilon} / 2$, which goes to zero as $\eta \rightarrow 0$. 
Now a straightforward computation (using the first 3 derivatives of the flow generated by $\mathbf{u}$ ) gives the result

$$
\mathbf{x}_{\eta}(t)-\mathbf{x}(t)=\boldsymbol{\xi}(t)+O\left(|\xi|^{2}\right),
$$

which we have argued is small with high probability in the limit $\eta \rightarrow 0$.

\section{References}

Adler, R.L., Konheim, A.G., McAndrew, M.H.: Topological entropy. Trans. Am. Math. Soc. 114, 309-319 (1965)

Arnol'd, V.I., Zel'dovich, Ya.B., Ruzmaikin, A.A., Sokoloff, D.D.: A magnetic field in a stationary flow with stretching in Riemannian space. Soviet Phys. JETP 54, 1083-1086 (1981)

Bayly, B.J.: Fast magnetic dynamos in chaotic flows. Phys. Rev. Lett. 57, 2800-2803 (1986)

Bayly, B.J.: Childress, S.: Construction of fast dynamos using unsteady flows and maps in three dimensions. Geophys. Astrophys. Fluid Dyn. 44, 207-240 (1988)

Bowen, R.E.: Entropy for group endomorphisms and homogeneous spaces. Trans. Am. Math. Soc. 153, 401-414 (1971)

Chorin, A.J., Hughes, T.J.R., McCracken, M.F., Marsden, J.E.: Product for mulas and numerical algorithms. Comm. Pure Appl. Math. 31, 205-256 (1978)

Collet, P.: Personal communication, 1992

Feller, W.: An Introduction to Probability Theory and Its Applications. New York: John Wiley, 1957

Finn, J.M., Ott, E.: Chaotic flows and fast magnetic dynamos. Phys. Fluids 31, 2992-3011 (1988)

Gilbert, A.D.: Fast dynamo action in the Ponomerenko dynamo. Geophys. Astrophys. Fluid Dyn. 44, 214-258 (1988)

Gilbert, A.D.: Towards a realistic fast dynamo: Models based on cat maps and pseudo-Anosov maps. Proc. Roy. Soc. Lond. 443, 585-606 (1993)

Gilbert, A.D., Bayly, B.J.: Magnetic field intermittency and fast dynamo action in random helical flows. J. Fluid Mech. 241, 199-214 (1992)

Hollerbach, R., Galloway, D.J., Proctor, M.R.E.: Numerical evidence of fast dynamo action in a spherical shell. Phys. Rev. Lett. 74, 3145-3148 (1995)

Katok, A.: Lyapunov exponents, entropy and periodic points for diffeomorphisms. Publ. Math. I.H.E.S. 51, 137-174 (1980)

Kifer, Yu.: Ergodic Theory of Random Transformations. Boston: Birkhäuser, 1986

Kifer, Yu., Yomdin, Y.: Volume growth and topological entropy for random transformations. In: Dynamical Systems, Lecture Notes in Mathematics No. 1342. Berlin, Heidelberg, New York: Springer, 1988

Klapper, I.: A study of fast dynamo action in chaotic helical cells. J. Fluid Mech. 239, 359-381 (1992)

Klapper, I.: Shadowing and the diffusionless limit in fast dynamo theory. Nonlinearity 6, 869-884 (1993)

Kunita, H.: Stochastic Flows and Stochastic Differential Equations. Cambridge: Cambridge University Press, 1990

Molchanov, S.A., Ruzmaikin, A.A., Sokolov, D.D.: Kinematic dynamo action in a random flow. Sov. Phys. Usp. 28, 307-326 (1985)

Newhouse, S.E.: Entropy and volume. Ergod. Th. \& Dynam. Sys. 8, 283-299 (1988)

Oseledets, V.: A multiplicative ergodic theorem. Lyapunov characteristic numbers for dynamical systems. Trans. Moscow Math. Soc. 19, 197-231 (1968)

Oseledets, V.: Fast dynamo problem for a smooth map on two-torus. Geophys. Astrophys. Fluid Dyn. 73, 133-146 (1993)

Pesin, Ya.B.: Families of invariant manifolds corresponding to non-zero characteristic exponents. Math. of the USSR, Izvestjia 10, 1261-1305 (1978)

Roberts, P.H.: An Introduction to Magnetohydrodynamics. London: Longmans, Green and Co., 1967

Ruelle, D.: An inequality of the entropy of differentiable maps. Bol. Sc. Bra. Mat. 9, 83-87 (1978) 
Soward, A.M.: An asymptotic solution of a fast dynamo in a two-dimensional pulsed flow. J. Fluid Mech. 180, 267-295 (1987)

Soward, A.M.: On the role of stagnation points and periodic particle paths in a two-dimensional pulsed flow fast dynamo model. Physica D 76, 181-201 (1994)

Vainshtein S.I., Zel'dovich, Ya.B.: Origin of magnetic fields in astrophysics. Sov. Phys. Usp. 15, 159-172 (1972)

Vishik, M.M.: Magnetic field generation by the motion of a highly conducting fluid. Geophys. Astrophys. Fluid Dyn. 48, 151-167 (1989)

Vishik, M.M.: Personal communication, 1992

Walters, P.: An Introduction to Ergodic Theory. Berlin, Heidelberg, New York: Springer, 1982

Yomdin, Y.: Volume growth and entropy. Israel J. Math. 57, 285-300 (1987)

Young, L.S.: Ergodic theory of differentiable dynamical systems. To appear in B. Branner, P. Hjorth (eds.) Real and Complex Dynamical Systems. Dordercht: Kluwer, 1995

Zel'dovich, Ya.B.: The magnetic field in the two-dimensional motion of a conducting turbulent fluid. Sov. Phys. JETP 4, 460-462 (1957)

Zel'dovich, Ya.B., Molchanov, S.A., Ruzmaikin, A.A., Sokolov, D.D.: Intermittency, diffusion and generation in a nonsteady random medium. Sov. Sci. Rev. C. Math. Phys. 7, 1-110 (1988)

Communicated by J.-P. Eckmann 